\title{
INFEASIBLE-INTERIOR-POINT PRIMAL-DUAL POTENTIAL-REDUCTION ALGORITHMS FOR LINEAR PROGRAMMING *
}

\author{
SHINJI MIZUNO ${ }^{\dagger}$, MASAKAZU KOJIMA ${ }^{\ddagger}$, AND MICHAEL J. TODD $§$
}

\begin{abstract}
In this paper, we propose primal-dual potential-reduction algorithms which can start from an infeasible interior point. We first describe two such algorithms and show that both are polynomial-time bounded. One of the algorithms decreases the Tanabe-Todd-Ye primal-dual potential function by a constant at each iteration under the condition that the duality gap decreases by at most the same ratio as the infeasibility. The other reduces a new potential function, which has one more term in the Tanabe-Todd-Ye potential function, by a fixed constant at each iteration without any other conditions on the step size. Finally, we describe modifications of these methods (incorporating centering steps) which dramatically decrease their computational complexity. Our algorithms also extend to the case of monotone linear complementarity problems.
\end{abstract}

Key words. Polynomial Time, Linear Programming, Primal-Dual, Infeasible-Interior-Point Algorithm, Potential Function.

AMS subject classifications. $90 \mathrm{C} 05,65 \mathrm{~K} 05$

1. Introduction. The primal-dual infeasible-interior-point algorithm for linear programming is a simple variant of the primal-dual (feasible-)interior-point algorithms developed by Megiddo [11], Kojima, Mizuno, and Yoshise [4, 5], Monteiro and Adler $[15,16]$, and Tanabe [18]. The algorithm can start from an infeasible point, while interior-point algorithms have to start from a feasible point. When we solve a given problem by an interior-point algorithm, we need to construct an artificial problem to get an initial feasible point. The advantage of the infeasible-interior-point algorithm over the interior-point algorithm is in solving the given problem directly. (This is a very significant advantage in practice. In theory, the complexity analysis of most of these methods still requires initial solutions which may need to have very large (big M) components.) The algorithm has been studied by Lustig [8], Lustig, Marsten, and Shanno [9], Marsten, Subramanian, Saltzman, Lustig, and Shanno [10], Tanabe [19], etc., and is known to be one of the most efficient interior-point algorithms (see for example [9, 10]). Kojima, Megiddo and Mizuno [3] demonstrated the global convergence of an infeasible-interior-point algorithm. Then Zhang [22] Mizuno [12], and Potra [17] proved polynomial-time convergence of certain infeasible-interior-point algorithms. Those algorithms generate a sequence of points in a neighborhood of the path of centers and they are classified as path-following algorithm.

In the framework of interior-point algorithms, potential functions have played important roles in determining a step size at each iteration and in obtaining a theoretical upper bound on the number of iterations (see Karmarkar [2], Ye [20], Ko-

* Copyright (C) by the Society for Industrial and Applied Mathematics, in SIAM Journal on Optimization, 5 (1995), pp. 52-67.

$\dagger$ The Institute of Statistical Mathematics, 4-6-7 Minami-Azabu, Minato-ku, Tokyo 106, Japan. Research performed while visiting Cornell University (April, 1992 - January, 1993) as an overseas research scholar of The Ministry of Science, Education and Culture of Japan.

¥ Department of Information Sciences, Tokyo Institute of Technology, 2-12-1 Oh-Okayama, Meguro, Tokyo 152, Japan. Research partially performed while visiting Cornell University (July, 1992).

$\S$ School of Operations Research and Industrial Engineering, Cornell University, Ithaca, New York 14853, USA. Research supported in part by NSF, AFOSR and ONR through NSF Grant DMS8920550 . 
jima, Mizuno, and Yoshise [6] etc.). Kojima, Noma, and Yoshise [7] investigated the global convergence of infeasible-interior-point (both potential-reduction and pathfollowing) algorithms for monotone complementarity problems. In this paper, we propose polynomial-time primal-dual potential-reduction algorithms which start from an infeasible interior point.

Let $\boldsymbol{A}$ be an $m \times n$ matrix, $\boldsymbol{b} \in R^{m}$, and $c \in R^{n}$. Consider the standard form linear program

$$
\begin{array}{ll}
\text { (P) Minimize } & \boldsymbol{c}^{T} \boldsymbol{x} \\
\text { subject to } & \boldsymbol{A} \boldsymbol{x}=\boldsymbol{b}, \boldsymbol{x} \geq \mathbf{0}
\end{array}
$$

and its dual

$$
\begin{array}{ll}
\text { (D) Maximize } & \boldsymbol{b}^{T} \boldsymbol{y} \\
\text { subject to } & \boldsymbol{A}^{T} \boldsymbol{y}+\boldsymbol{z}=\boldsymbol{c}, \boldsymbol{z} \geq \mathbf{0} .
\end{array}
$$

We assume that the matrix $\boldsymbol{A}$ has full row rank, i.e., $\operatorname{rank} \boldsymbol{A}=m$. We call $(\boldsymbol{x}, \boldsymbol{y}, \boldsymbol{z})$ an (infeasible) interior point if $\boldsymbol{x}>\mathbf{0}$ and $\boldsymbol{z}>\mathbf{0}$, and a feasible interior point if in addition $\boldsymbol{A x}=\boldsymbol{b}$ and $\boldsymbol{A}^{T} \boldsymbol{y}+\boldsymbol{z}=\boldsymbol{c}$.

Let $\left(\boldsymbol{x}^{1}, \boldsymbol{y}^{1}, \boldsymbol{z}^{1}\right)$ be an interior point and $\sigma>0$ be such that $\left(\boldsymbol{x}^{1}\right)^{T} \boldsymbol{z}^{1}$ $>\sigma\left\|\left(\boldsymbol{A} \boldsymbol{x}^{1}-\boldsymbol{b}, \boldsymbol{A}^{T} \boldsymbol{y}^{1}+\boldsymbol{z}^{1}-\boldsymbol{c}\right)\right\|$, where $\|\cdot\|$ denotes the $\ell_{2}$-norm. For a constant $\nu \geq 0$, we define two primal-dual potential functions:

$$
\begin{aligned}
\phi(\boldsymbol{x}, \boldsymbol{z}):= & (n+\nu) \ln \left(\boldsymbol{x}^{T} \boldsymbol{z}\right)-\sum_{i=1}^{n} \ln \left(x_{i} z_{i}\right)-n \ln n, \\
\psi(\boldsymbol{x}, \boldsymbol{y}, \boldsymbol{z}):= & (n+\nu+1) \ln \left(\boldsymbol{x}^{T} \boldsymbol{z}\right)-\sum_{i=1}^{n} \ln \left(x_{i} z_{i}\right)-n \ln n \\
& -\ln \left(\boldsymbol{x}^{T} \boldsymbol{z}-\sigma\left\|\left(\boldsymbol{A} \boldsymbol{x}-\boldsymbol{b}, \boldsymbol{A}^{T} \boldsymbol{y}+\boldsymbol{z}-\boldsymbol{c}\right)\right\|\right) .
\end{aligned}
$$

The first is known as the Tanabe-Todd-Ye primal-dual potential function (used for feasible-interior-point algorithms) and the second is defined here for an infeasibleinterior-point algorithm. If $(\boldsymbol{x}, \boldsymbol{y}, \boldsymbol{z})$ is feasible, $\psi(\boldsymbol{x}, \boldsymbol{y}, \boldsymbol{z})=\phi(\boldsymbol{x}, \boldsymbol{z})$. Note that $\psi$ involves the norm of a vector formed from the primal and dual infeasibilities. It appears that this would be very sensitive to different scalings of the original problem. However, we shall see in (6) below that each component of this vector decreases at the same rate during the algorithms. Hence the norm measures how much each infeasibility has been reduced.

Sections 2 through 4 of this paper construct two infeasible-interior-point algorithms, namely Algorithms I and II, which start from the initial point $\left(\boldsymbol{x}^{1}, \boldsymbol{y}^{1}, \boldsymbol{z}^{1}\right)$ and generate a sequence $\left\{\left(\boldsymbol{x}^{k}, \boldsymbol{y}^{k}, \boldsymbol{z}^{k}\right)\right\}$ of interior points. Algorithms I and II decrease the potential functions $\phi$ and $\psi$ at each iteration, respectively. The step size $\alpha$ at the $k$ th iterate $\left(\boldsymbol{x}^{k}, \boldsymbol{y}^{k}, \boldsymbol{z}^{k}\right)$ of Algorithm I is determined such that $\phi$ decreases at least a constant value and an extra condition holds, while Algorithm II does not need any such condition. So Algorithm I is a constrained potential-reduction algorithm, while Algorithm II is a pure potential-reduction algorithm. In the worst case, the decrease in the potential functions at each iteration is only $\Omega\left(n^{-2}\right)$, and this leads to a complexity bound of $O\left(n^{2.5} L\right)$ iterations, where $L$ is related to the initialization and the termination criterion of the algorithms. Then Section 5 describes variants 
that require only $O(n L)$ iterations by adding centering steps when the current iterate lies outside a wide neighborhood of the path of centers. The centering steps keep the "duality gap" and the infeasibilities fixed while decreasing the potential functions $\phi$ and $\psi$. Finally, Section 6 contains a discussion of why the complexity bounds of these infeasible-interior-point methods are so much higher than those for feasibleinterior-point algorithms, and shows how the algorithms also extend to monotone linear complementarity problems. We chose to confine ourselves to the more familiar setting of linear programming for the main development.

2. A constrained potential-reduction algorithm. The path of centers consists of the solutions $(\boldsymbol{x}, \boldsymbol{y}, \boldsymbol{z})$ to the system of equations

$$
\left(\begin{array}{c}
\boldsymbol{A} \boldsymbol{x}-\boldsymbol{b} \\
\boldsymbol{A}^{T} \boldsymbol{y}+\boldsymbol{z}-\boldsymbol{c} \\
\boldsymbol{X} \boldsymbol{z}-\mu \boldsymbol{e}
\end{array}\right)=\mathbf{0}
$$

for all $\mu>0$. Here $\boldsymbol{X}:=\operatorname{diag}(\boldsymbol{x})$ denotes the $n \times n$ diagonal matrix containing the coordinates of a vector $\boldsymbol{x} \in R^{n}$ and $\boldsymbol{e}:=(1, \ldots, 1)^{T} \in R^{n}$. At each iteration, we assign the value $\left(\boldsymbol{x}^{k}\right)^{T} \boldsymbol{z}^{k} /(n+\nu)$ to the parameter $\mu$, and then compute the Newton direction $(\boldsymbol{\Delta} \boldsymbol{x}, \boldsymbol{\Delta} \boldsymbol{y}, \boldsymbol{\Delta} \boldsymbol{z})$ at $\left(\boldsymbol{x}^{k}, \boldsymbol{y}^{k}, \boldsymbol{z}^{k}\right)$ for the system (1) of equations; that is, $(\boldsymbol{\Delta} \boldsymbol{x}, \boldsymbol{\Delta} \boldsymbol{y}, \boldsymbol{\Delta} \boldsymbol{z})$ is the unique solution of the system of linear equations

$$
\left(\begin{array}{ccc}
\boldsymbol{A} & \mathbf{0} & \mathbf{0} \\
\mathbf{0} & \boldsymbol{A}^{T} & \boldsymbol{I} \\
\boldsymbol{Z}^{k} & \mathbf{0} & \boldsymbol{X}^{k}
\end{array}\right)\left(\begin{array}{c}
\Delta \boldsymbol{x} \\
\boldsymbol{\Delta} \boldsymbol{y} \\
\boldsymbol{\Delta} \boldsymbol{z}
\end{array}\right)=-\left(\begin{array}{c}
\boldsymbol{A} \boldsymbol{x}^{k}-\boldsymbol{b} \\
\boldsymbol{A}^{T} \boldsymbol{y}^{k}+\boldsymbol{z}^{k}-\boldsymbol{c} \\
\boldsymbol{X}^{k} \boldsymbol{z}^{k}-\mu \boldsymbol{e}
\end{array}\right)
$$

where $\boldsymbol{X}^{k}:=\operatorname{diag}\left(\boldsymbol{x}^{k}\right)$ and $\boldsymbol{Z}^{k}:=\operatorname{diag}\left(\boldsymbol{z}^{k}\right)$.

Let $\rho$ be a positive constant for which we want to find the optimal solutions $x^{*}$ of (P) and $\left(\boldsymbol{y}^{*}, \boldsymbol{z}^{*}\right)$ of (D), if they exist, such that

$$
\left\|\left(\boldsymbol{x}^{*}, \boldsymbol{z}^{*}\right)\right\|_{\infty} \leq \rho .
$$

\section{Algorithm I}

Step 1: Choose $\gamma_{0} \in(0,1]$ and a positive constant $\delta$ (which may depend on $n$ and $\nu)$. Set $\left(\boldsymbol{x}^{1}, \boldsymbol{y}^{1}, \boldsymbol{z}^{1}\right):=\gamma_{0} \rho(\boldsymbol{e}, \mathbf{0}, \boldsymbol{e})$. Let $k:=1$.

Step 2: If $\left(\boldsymbol{x}^{k}\right)^{T} \boldsymbol{z}^{k} \leq \epsilon$ then stop.

Step 3: Let $\mu:=\left(\boldsymbol{x}^{\bar{k}}\right)^{T} \boldsymbol{z}^{k} /(n+\nu)$. Compute the solution $(\boldsymbol{\Delta} \boldsymbol{x}, \boldsymbol{\Delta} \boldsymbol{y}, \boldsymbol{\Delta} \boldsymbol{z})$ at $\left(\boldsymbol{x}^{k}, \boldsymbol{y}^{k}, \boldsymbol{z}^{k}\right)$ of the system (2) of equations.

Step 4: Find a step size $\alpha$ such that

$$
\begin{aligned}
\phi\left(\boldsymbol{x}^{k}+\alpha \boldsymbol{\Delta} \boldsymbol{x}, \boldsymbol{z}^{k}+\alpha \boldsymbol{\Delta} \boldsymbol{z}\right) & \leq \phi\left(\boldsymbol{x}^{k}, \boldsymbol{z}^{k}\right)-\delta \\
\left(\boldsymbol{x}^{k}+\alpha \boldsymbol{\Delta} \boldsymbol{x}\right)^{T}\left(\boldsymbol{z}^{k}+\alpha \boldsymbol{\Delta} \boldsymbol{z}\right) & \geq(1-\alpha)\left(\boldsymbol{x}^{k}\right)^{T} \boldsymbol{z}^{k} .
\end{aligned}
$$

If we cannot find such a step size then stop.

Step 5: Let $\left(\boldsymbol{x}^{k+1}, \boldsymbol{y}^{k+1}, \boldsymbol{z}^{k+1}\right):=\left(\boldsymbol{x}^{k}, \boldsymbol{y}^{k}, \boldsymbol{z}^{k}\right)+\alpha(\boldsymbol{\Delta} \boldsymbol{x}, \boldsymbol{\Delta} \boldsymbol{y}, \boldsymbol{\Delta} \boldsymbol{z})$. Increase $k$ by 1 and go to Step 2 .

The direction $(\boldsymbol{\Delta} \boldsymbol{x}, \boldsymbol{\Delta} \boldsymbol{y}, \boldsymbol{\Delta} \boldsymbol{z})$ is, except for the choice of $\mu$, the same as in the earlier primal-dual infeasible-interior-point algorithms. If the current iterate is feasible, our choice of $\mu$ yields the direction that is the projected scaled steepest descent direction for the potential function $\phi$ (or $\psi$ ) [6]. 
Since $\phi(\boldsymbol{x}, \boldsymbol{z}) \geq \nu \ln \left(\boldsymbol{x}^{T} \boldsymbol{z}\right)$ and the potential function decreases by a constant $\delta$ at each iteration, Algorithm I terminates in $O(\nu L / \delta)$ iterations provided that $\phi\left(\boldsymbol{x}^{1}, \boldsymbol{z}^{1}\right)=$ $O(\nu L)$ and $\ln (1 / \epsilon)=O(L)$. If $L \geq \ln n$ and $\ln \rho=O(L)$ then $\phi\left(\boldsymbol{x}^{1}, \boldsymbol{z}^{1}\right)=O(\nu L)$. In the next section, we show that if there are optimal solutions $\boldsymbol{x}^{*}$ of $(\mathrm{P})$ and $\left(\boldsymbol{y}^{*}, \boldsymbol{z}^{*}\right)$ of (D) such that $\left\|\left(\boldsymbol{x}^{*}, \boldsymbol{z}^{*}\right)\right\|_{\infty} \leq \rho$, then there exists a step size $\alpha$ which satisfies (3) and (4) for $\delta:=\gamma_{0}^{4} /\left(300(n+\nu)^{2}\right)$. (Condition (4) is what makes this a constrained potential-reduction algorithm.) Hence we have the following result.

THEOREM 1. Let $L \geq \ln n$ ( $L$ may be the input size of problem $(P))$ and $\gamma_{0} \in$ $(0,1]$. Suppose that $\ln \rho=O(L), \ln (1 / \epsilon)=O(L), \nu \geq \sqrt{n}$, and $\delta:=\gamma_{0}^{4} /\left(300(n+\nu)^{2}\right)$. Then Algorithm I terminates in $O\left(\nu(n+\nu)^{2} L\right)$ iterations. If the algorithm stops in Step 2, we get an approximate solution; otherwise (if it stops in Step 4) there are no optimal solutions $\boldsymbol{x}^{*}$ of $(P)$ and $\left(\boldsymbol{y}^{*}, \boldsymbol{z}^{*}\right)$ of $(D)$ such that $\left\|\left(\boldsymbol{x}^{*}, \boldsymbol{z}^{*}\right)\right\|_{\infty} \leq \rho$.

3. Analysis of Algorithm I. Theorem 1 follows from the following four lemmas. The first gives a bound on the decrease in $\phi$.

Lemma 2. (Kojima, Mizuno, and Yoshise [6]) For any n-vectors $\boldsymbol{x}>\mathbf{0}, \boldsymbol{z}>\mathbf{0}$, $\boldsymbol{\Delta} \boldsymbol{x}, \boldsymbol{\Delta} \boldsymbol{z}$, and $\alpha>0$ such that $\left\|\alpha \boldsymbol{X}^{-1} \boldsymbol{\Delta} \boldsymbol{x}\right\|_{\infty} \leq \tau$ and $\left\|\alpha \boldsymbol{Z}^{-1} \boldsymbol{\Delta} \boldsymbol{z}\right\|_{\infty} \leq \tau$ for a constant $\tau \in(0,1)$, we have

$$
\begin{aligned}
\phi(\boldsymbol{x}+\alpha \boldsymbol{\Delta} \boldsymbol{x}, \boldsymbol{z}+\alpha \boldsymbol{\Delta} \boldsymbol{z}) \leq & \phi(\boldsymbol{x}, \boldsymbol{z})+\left(\frac{n+\nu}{\boldsymbol{x}^{T} \boldsymbol{z}} \boldsymbol{e}-(\boldsymbol{X} \boldsymbol{Z})^{-1} \boldsymbol{e}\right)^{T}(\boldsymbol{Z} \boldsymbol{\Delta} \boldsymbol{x}+\boldsymbol{X} \boldsymbol{\Delta} \boldsymbol{z}) \alpha \\
& +\left((n+\nu) \frac{\boldsymbol{\Delta}^{T} \boldsymbol{\Delta} \boldsymbol{z}}{\boldsymbol{x}^{T} \boldsymbol{z}}+\frac{\left\|\boldsymbol{X}^{-1} \boldsymbol{\Delta} \boldsymbol{x}\right\|^{2}+\left\|\boldsymbol{Z}^{-1} \boldsymbol{\Delta} \boldsymbol{z}\right\|^{2}}{2(1-\tau)}\right) \alpha^{2} .
\end{aligned}
$$

The next result is important in analyzing the linear term above, with $\boldsymbol{v}:=\boldsymbol{X}^{1 / 2} \boldsymbol{Z}^{1 / 2} \boldsymbol{e}$.

Lemma 3. (Lemma 2.5 in Kojima, Mizuno, and Yoshise [6]) For any n-vector $\boldsymbol{v}>\mathbf{0}$ and $\nu \geq \sqrt{n}$

$$
\left\|\boldsymbol{V}^{-1} e-\frac{n+\nu}{\boldsymbol{v}^{T} \boldsymbol{v}} \boldsymbol{v}\right\| \geq \frac{\sqrt{3}}{2 v_{\min }}
$$

where $\boldsymbol{V}:=\operatorname{diag}(\boldsymbol{v})$ and $v_{\min }:=\min _{i} v_{i}$.

Note that $\nu=\sqrt{n}$ is fixed in [6], but the proof is valid for any $\nu \geq \sqrt{n}$.

Let $\alpha^{k}$ be the step size at the $k$ th iteration of Algorithm I. We define a sequence $\left\{\theta^{k}\right\}$ by

$$
\theta^{1}:=1 \text { and } \theta^{k+1}:=\left(1-\alpha^{k}\right) \theta^{k} \text { for } k=1,2,3, \cdots .
$$

As shown in [3], we have

$$
\left(\boldsymbol{A} \boldsymbol{x}^{k}-\boldsymbol{b}, \boldsymbol{A}^{T} \boldsymbol{y}^{k}+\boldsymbol{z}^{k}-\boldsymbol{c}\right)=\theta^{k}\left(\boldsymbol{A} \boldsymbol{x}^{1}-\boldsymbol{b}, \boldsymbol{A}^{T} \boldsymbol{y}^{1}+\boldsymbol{z}^{1}-\boldsymbol{c}\right) .
$$

The following result is used to bound the second-order term in (5). The parameter $\gamma_{1}$ is introduced to allow this lemma to be used in the analysis of Algorithm II also.

Lemma 4. (based on Mizuno [12]) Let $\gamma_{0} \in(0,1], \gamma_{1} \in(0,1]$, and $\rho>0$. Suppose that

$$
\begin{aligned}
\left(\boldsymbol{x}^{1}, \boldsymbol{y}^{1}, \boldsymbol{z}^{1}\right) & =\gamma_{0} \rho(\boldsymbol{e}, \mathbf{0}, \boldsymbol{e}) \\
\left(\boldsymbol{A} \boldsymbol{x}^{k}-\boldsymbol{b}, \boldsymbol{A}^{T} \boldsymbol{y}^{k}+\boldsymbol{z}^{k}-\boldsymbol{c}\right) & =\theta^{k}\left(\boldsymbol{A} \boldsymbol{x}^{1}-\boldsymbol{b}, \boldsymbol{A}^{T} \boldsymbol{y}^{1}+\boldsymbol{z}^{1}-\boldsymbol{c}\right) \\
\left(\boldsymbol{x}^{k}\right)^{T} \boldsymbol{z}^{k} & \geq \theta^{k} \gamma_{1}\left(\boldsymbol{x}^{1}\right)^{T} \boldsymbol{z}^{1}
\end{aligned}
$$


If there exist optimal solutions $\boldsymbol{x}^{*}$ of $(P)$ and $\left(\boldsymbol{y}^{*}, \boldsymbol{z}^{*}\right)$ of $(D)$ such that $\left\|\left(\boldsymbol{x}^{*}, \boldsymbol{z}^{*}\right)\right\|_{\infty} \leq \rho$ then we have

$$
\begin{aligned}
\left\|\boldsymbol{D}^{-1} \boldsymbol{\Delta} \boldsymbol{x}\right\| & \leq \frac{5}{\gamma_{0}^{2} \gamma_{1}} \frac{\left(\boldsymbol{x}^{k}\right)^{T} \boldsymbol{z}^{k}}{v_{\min }} \\
\|\boldsymbol{D} \boldsymbol{\Delta} \boldsymbol{z}\| & \leq \frac{5}{\gamma_{0}^{2} \gamma_{1}} \frac{\left(\boldsymbol{x}^{k}\right)^{T} \boldsymbol{z}^{k}}{v_{\min }}
\end{aligned}
$$

where $\boldsymbol{D}:=\left(\boldsymbol{X}^{k}\right)^{1 / 2}\left(\boldsymbol{Z}^{k}\right)^{-1 / 2}$ and $v_{\min }:=\min _{i} \sqrt{x_{i}^{k} z_{i}^{k}}$.

Proof: Assume that there exist optimal solutions $\boldsymbol{x}^{*}$ of $(\mathrm{P})$ and $\left(\boldsymbol{y}^{*}, \boldsymbol{z}^{*}\right)$ of (D) such that $\left\|\left(\boldsymbol{x}^{*}, \boldsymbol{z}^{*}\right)\right\|_{\infty} \leq \rho$. Then we have

$$
\left(\theta^{k} \boldsymbol{x}^{1}+\left(1-\theta^{k}\right) \boldsymbol{x}^{*}-\boldsymbol{x}^{k}\right)^{T}\left(\theta^{k} \boldsymbol{z}^{1}+\left(1-\theta^{k}\right) \boldsymbol{z}^{*}-\boldsymbol{z}^{k}\right)=0,
$$

which implies

$\left(\theta^{k} \boldsymbol{x}^{1}+\left(1-\theta^{k}\right) \boldsymbol{x}^{*}\right)^{T} \boldsymbol{z}^{k}+\left(\theta^{k} \boldsymbol{z}^{1}+\left(1-\theta^{k}\right) \boldsymbol{z}^{*}\right)^{T} \boldsymbol{x}^{k}=\left(\theta^{k} \boldsymbol{x}^{1}+\left(1-\theta^{k}\right) \boldsymbol{x}^{*}\right)^{T}\left(\theta^{k} \boldsymbol{z}^{1}+\left(1-\theta^{k}\right) \boldsymbol{z}^{*}\right)+\left(\boldsymbol{x}^{k}\right)^{T} \boldsymbol{z}^{k}$.

By using this equality, $\boldsymbol{x}^{1}=\boldsymbol{z}^{1}=\gamma_{0} \rho \boldsymbol{e}, \boldsymbol{x}^{*} \leq \rho \boldsymbol{e}, \boldsymbol{z}^{*} \leq \rho \boldsymbol{e}$, and $x_{i}^{*} z_{i}^{*}=0$ for each $i$, we have

$$
\begin{aligned}
\theta^{k}\left(\gamma_{0} \rho\right)\left\|\left(\boldsymbol{x}^{k}, \boldsymbol{z}^{k}\right)\right\|_{1} & =\theta^{k}\left(\left(\boldsymbol{z}^{1}\right)^{T} \boldsymbol{x}^{k}+\left(\boldsymbol{x}^{1}\right)^{T} \boldsymbol{z}^{k}\right) \\
& \leq\left(\theta^{k} \boldsymbol{x}^{1}+\left(1-\theta^{k}\right) \boldsymbol{x}^{*}\right)^{T} \boldsymbol{z}^{k}+\left(\theta^{k} \boldsymbol{z}^{1}+\left(1-\theta^{k}\right) \boldsymbol{z}^{*}\right)^{T} \boldsymbol{x}^{k} \\
& =\left(\theta^{k} \boldsymbol{x}^{1}+\left(1-\theta^{k}\right) \boldsymbol{x}^{*}\right)^{T}\left(\theta^{k} \boldsymbol{z}^{1}+\left(1-\theta^{k}\right) \boldsymbol{z}^{*}\right)+\left(\boldsymbol{x}^{k}\right)^{T} \boldsymbol{z}^{k} \\
& \leq n \theta^{k} \gamma_{0} \rho^{2}+\left(\boldsymbol{x}^{k}\right)^{T} \boldsymbol{z}^{k}
\end{aligned}
$$

where the last inequality follows from the fact that for each $i$ one of $\theta^{k} x_{i}^{1}+\left(1-\theta^{k}\right) x_{i}^{*}$ and $\theta^{k} z_{i}^{1}+\left(1-\theta^{k}\right) z_{i}^{*}$ is at most $\theta^{k} \gamma_{0} \rho$ and the other is at most $\rho$. From $(7),\left(\boldsymbol{x}^{k}\right)^{T} \boldsymbol{z}^{k} \geq$ $\theta^{k} \gamma_{1}\left(\boldsymbol{x}^{1}\right)^{T} \boldsymbol{z}^{1}=n \theta^{k} \gamma_{0}^{2} \gamma_{1} \rho^{2}$. Hence we have

$$
\theta^{k} \gamma_{0} \rho\left\|\left(\boldsymbol{x}^{k}, \boldsymbol{z}^{k}\right)\right\|_{1} \leq \frac{2}{\gamma_{0} \gamma_{1}}\left(\boldsymbol{x}^{k}\right)^{T} \boldsymbol{z}^{k}
$$

From (2) and (6), we get

$$
\begin{aligned}
& \left(\begin{array}{ccc}
\boldsymbol{A} & \mathbf{0} & \mathbf{0} \\
\mathbf{0} & \boldsymbol{A}^{T} & \boldsymbol{I} \\
\boldsymbol{Z}^{k} & \mathbf{0} & \boldsymbol{X}^{k}
\end{array}\right)\left(\begin{array}{c}
\boldsymbol{\Delta} \boldsymbol{x}+\theta^{k}\left(\boldsymbol{x}^{1}-\boldsymbol{x}^{*}\right) \\
\boldsymbol{\Delta} \boldsymbol{y}+\theta^{k}\left(\boldsymbol{y}^{1}-\boldsymbol{y}^{*}\right) \\
\boldsymbol{\Delta} \boldsymbol{z}+\theta^{k}\left(\boldsymbol{z}^{1}-\boldsymbol{z}^{*}\right)
\end{array}\right) \\
& =-\left(\begin{array}{c}
\mathbf{0} \\
\mathbf{0} \\
\boldsymbol{X}^{k} \boldsymbol{z}^{k}-\mu \boldsymbol{e}-\theta^{k} \boldsymbol{Z}^{k}\left(\boldsymbol{x}^{1}-\boldsymbol{x}^{*}\right)-\theta^{k} \boldsymbol{X}^{k}\left(\boldsymbol{z}^{1}-\boldsymbol{z}^{*}\right)
\end{array}\right) .
\end{aligned}
$$

Then we have via a straightforward computation (see also Mizuno [12])

$$
\begin{aligned}
\boldsymbol{D}^{-1} \boldsymbol{\Delta} \boldsymbol{x}= & -\theta^{k} \boldsymbol{Q} \boldsymbol{D}^{-1}\left(\boldsymbol{x}^{1}-\boldsymbol{x}^{*}\right)+\theta^{k}(\boldsymbol{I}-\boldsymbol{Q}) \boldsymbol{D}\left(\boldsymbol{z}^{1}-\boldsymbol{z}^{*}\right) \\
& -(\boldsymbol{I}-\boldsymbol{Q})\left(\boldsymbol{X}^{k} \boldsymbol{Z}^{k}\right)^{-1 / 2}\left(\boldsymbol{X}^{k} \boldsymbol{z}^{k}-\mu \boldsymbol{e}\right),
\end{aligned}
$$

where $\boldsymbol{Q}:=\boldsymbol{D} \boldsymbol{A}^{T}\left(\boldsymbol{A} \boldsymbol{D}^{2} \boldsymbol{A}^{T}\right)^{-1} \boldsymbol{A} \boldsymbol{D}$. Since $\boldsymbol{Q}$ and $\boldsymbol{I}-\boldsymbol{Q}$ are orthogonal projections, we have

$$
\left\|\boldsymbol{D}^{-1} \boldsymbol{\Delta} \boldsymbol{x}\right\| \leq \theta^{k}\left\|\boldsymbol{D}^{-1}\left(\boldsymbol{x}^{1}-\boldsymbol{x}^{*}\right)\right\|+\theta^{k}\left\|\boldsymbol{D}\left(\boldsymbol{z}^{1}-\boldsymbol{z}^{*}\right)\right\|+\left\|\left(\boldsymbol{X}^{k} \boldsymbol{Z}^{k}\right)^{-1 / 2}\left(\boldsymbol{X}^{k} \boldsymbol{z}^{k}-\mu \boldsymbol{e}\right)\right\| .
$$


From the definition of $\rho,-\rho \boldsymbol{e} \leq \boldsymbol{x}^{1}-\boldsymbol{x}^{*} \leq \rho \boldsymbol{e}$ and $-\rho \boldsymbol{e} \leq \boldsymbol{z}^{1}-\boldsymbol{z}^{*} \leq \rho \boldsymbol{e}$. Thus we have

$$
\begin{aligned}
\left\|\boldsymbol{D}^{-1} \boldsymbol{\Delta} \boldsymbol{x}\right\| & \leq \theta^{k} \rho\left\|\boldsymbol{D}^{-1} \boldsymbol{e}\right\|+\theta^{k} \rho\|\boldsymbol{D} \boldsymbol{e}\|+\left\|\left(\boldsymbol{X}^{k} \boldsymbol{Z}^{k}\right)^{-1 / 2}\left(\boldsymbol{X}^{k} \boldsymbol{z}^{k}-\mu \boldsymbol{e}\right)\right\| \\
& \leq \theta^{k} \rho\left\|\left(\boldsymbol{X}^{k} \boldsymbol{Z}^{k}\right)^{-1 / 2}\right\|\left(\left\|\boldsymbol{z}^{k}\right\|+\left\|\boldsymbol{x}^{k}\right\|\right)+\sqrt{\sum_{i=1}^{n}\left(\left(x_{i}^{k} z_{i}^{k}\right)^{1 / 2}-\mu\left(x_{i}^{k} z_{i}^{k}\right)^{-1 / 2}\right)^{2}} \\
(12) \leq & \leq \theta^{k} \rho\left\|\left(\boldsymbol{X}^{k} \boldsymbol{Z}^{k}\right)^{-1 / 2}\right\|\left\|\left(\boldsymbol{x}^{k}, \boldsymbol{z}^{k}\right)\right\|+\sqrt{\left(\boldsymbol{x}^{k}\right)^{T} \boldsymbol{z}^{k}-2 n \mu+\sum_{i=1}^{n} \mu^{2}\left(x_{i}^{k} z_{i}^{k}\right)^{-1} .}
\end{aligned}
$$

By using $v_{\text {min }}=\min _{i} \sqrt{x_{i}^{k} z_{i}^{k}}, \mu=\left(\boldsymbol{x}^{k}\right)^{T} \boldsymbol{z}^{k} /(n+\nu)$, and (from (9))

$$
\left\|\left(\boldsymbol{x}^{k}, \boldsymbol{z}^{k}\right)\right\| \leq\left\|\left(\boldsymbol{x}^{k}, \boldsymbol{z}^{k}\right)\right\|_{1} \leq \frac{2}{\gamma_{0}^{2} \gamma_{1} \theta^{k} \rho}\left(\boldsymbol{x}^{k}\right)^{T} \boldsymbol{z}^{k},
$$

we see

$$
\begin{aligned}
&\left\|\boldsymbol{D}^{-1} \boldsymbol{\Delta} \boldsymbol{x}\right\| \leq 2 \theta^{k} \rho \frac{1}{v_{\min }} \frac{2}{\gamma_{0}^{2} \gamma_{1} \theta^{k} \rho}\left(\boldsymbol{x}^{k}\right)^{T} \boldsymbol{z}^{k}+\sqrt{\left(\boldsymbol{x}^{k}\right)^{T} \boldsymbol{z}^{k}-2 \frac{n}{n+\nu}\left(\boldsymbol{x}^{k}\right)^{T} \boldsymbol{z}^{k}+\frac{n\left(\left(\boldsymbol{x}^{k}\right)^{T} \boldsymbol{z}^{k}\right)^{2}}{(n+\nu)^{2}\left(v_{m i n}\right)^{2}}} \\
&=\left(\frac{4}{\gamma_{0}^{2} \gamma_{1}}+\sqrt{\left(1-2 \frac{n}{n+\nu}\right) \frac{\left(v_{\min }\right)^{2}}{\left(\boldsymbol{x}^{k}\right)^{T} \boldsymbol{z}^{k}}+\frac{n}{(n+\nu)^{2}}}\right) \frac{\left(\boldsymbol{x}^{k}\right)^{T} \boldsymbol{z}^{k}}{v_{\min }} \\
&\left(14 £ \frac{5}{\gamma_{0}^{2} \gamma_{1}} \frac{\left(\boldsymbol{x}^{k}\right)^{T} \boldsymbol{z}^{k}}{v_{m i n}} .\right.
\end{aligned}
$$

The other inequality follows from a similar analysis of

$$
\begin{aligned}
\boldsymbol{D} \boldsymbol{\Delta} \boldsymbol{z}= & -\theta^{k}(\boldsymbol{I}-\boldsymbol{Q}) \boldsymbol{D}\left(\boldsymbol{z}^{1}-\boldsymbol{z}^{*}\right)+\theta^{k} \boldsymbol{Q} \boldsymbol{D}^{-1}\left(\boldsymbol{x}^{1}-\boldsymbol{x}^{*}\right) \\
& -\boldsymbol{Q}\left(\boldsymbol{X}^{k} \boldsymbol{Z}^{k}\right)^{-1 / 2}\left(\boldsymbol{X}^{k} \boldsymbol{z}^{k}-\mu \boldsymbol{e}\right) .
\end{aligned}
$$

Note that if (4) holds until the $(k-1)$ th iteration, then we have (7) for $\gamma_{1}=1$ by the definition of $\theta^{k}$. This also shows that if Algorithm I stops in Step 2, the infeasibility of $\boldsymbol{x}$ and $(\boldsymbol{y}, \boldsymbol{z})$ has been reduced at least as much as the duality gap, so we do have approximate solutions. Indeed, we have almost optimal solutions to a nearby linear programming problem and its dual.

Finally, the lemma below completes the proof of Theorem 1.

Lemma 5. Let $\nu \geq \sqrt{n}, \gamma_{0} \in(0,1], \gamma_{1} \in(0,1]$, and $\delta:=\frac{\gamma_{0}^{4} \gamma_{1}^{2}}{300(n+\nu)^{2}}$, and suppose (7) holds. If there exist optimal solutions $\boldsymbol{x}^{*}$ of $(P)$ and $\left(\boldsymbol{y}^{*}, \boldsymbol{z}^{*}\right)$ of $(D)$ such that $\left\|\left(\boldsymbol{x}^{*}, \boldsymbol{z}^{*}\right)\right\|_{\infty} \leq \rho$ then we have (3) and (4) for

$$
\alpha:=\frac{\gamma_{0}^{4} \gamma_{1}^{2} v_{\min }^{2}}{100(n+\nu)\left(\boldsymbol{x}^{k}\right)^{T} \boldsymbol{z}^{k}}
$$

at the kth iteration.

Proof: Let $\boldsymbol{v}:=\left(\boldsymbol{X}^{k} \boldsymbol{Z}^{k}\right)^{1 / 2} \boldsymbol{e}$ and $\boldsymbol{D}:=\left(\boldsymbol{X}^{k}\right)^{1 / 2}\left(\boldsymbol{Z}^{k}\right)^{-1 / 2}$. Then if $\tau$ satisfies the hypotheses of Lemma 2 , we have

$$
\phi\left(\boldsymbol{x}^{k}+\alpha \boldsymbol{\Delta} \boldsymbol{x}, \boldsymbol{z}^{k}+\alpha \boldsymbol{\Delta} \boldsymbol{z}\right)-\phi\left(\boldsymbol{x}^{k}, \boldsymbol{z}^{k}\right)
$$




$$
\begin{aligned}
\leq & \left(\frac{n+\nu}{\boldsymbol{v}^{T} \boldsymbol{v}} \boldsymbol{e}-\boldsymbol{V}^{-2} \boldsymbol{e}\right)^{T} \boldsymbol{V}\left(\boldsymbol{D}^{-1} \boldsymbol{\Delta} \boldsymbol{x}+\boldsymbol{D} \boldsymbol{\Delta} \boldsymbol{z}\right) \alpha \\
& +\left((n+\nu) \frac{\boldsymbol{\Delta} \boldsymbol{x}^{T} \boldsymbol{\Delta} \boldsymbol{z}}{\boldsymbol{v}^{T} \boldsymbol{v}}+\frac{\left\|\boldsymbol{V}^{-1} \boldsymbol{D}^{-1} \boldsymbol{\Delta} \boldsymbol{x}\right\|^{2}+\left\|\boldsymbol{V}^{-1} \boldsymbol{D} \boldsymbol{\Delta} \boldsymbol{z}\right\|^{2}}{2(1-\tau)}\right) \alpha^{2} .
\end{aligned}
$$

By the third equality in (2) with $\mu=\boldsymbol{v}^{T} \boldsymbol{v} /(n+\nu)$, we have

$$
D^{-1} \Delta x+D \Delta z=-\left(v-\frac{v^{T} v}{n+\nu} V^{-1} e\right) .
$$

From this equality, we also have, from

$$
\begin{gathered}
\left(\boldsymbol{D}^{-1} \boldsymbol{\Delta} \boldsymbol{x}\right)^{T}(\boldsymbol{D} \boldsymbol{\Delta} \boldsymbol{z})=\frac{1}{4}\left\{\left\|\boldsymbol{D}^{-1} \boldsymbol{\Delta} \boldsymbol{x}+\boldsymbol{D} \boldsymbol{\Delta} \boldsymbol{z}\right\|^{2}-\left\|\boldsymbol{D}^{-1} \boldsymbol{\Delta} \boldsymbol{x}-\boldsymbol{D} \boldsymbol{\Delta} \boldsymbol{z}\right\|^{2}\right\} \\
\boldsymbol{\Delta} \boldsymbol{x}^{T} \boldsymbol{\Delta} z \leq \frac{1}{4}\left\|\boldsymbol{v}-\frac{\boldsymbol{v}^{T} \boldsymbol{v}}{n+\nu} \boldsymbol{V}^{-1} \boldsymbol{e}\right\|^{2}
\end{gathered}
$$

By Lemma 4, we see that

$$
\begin{aligned}
\left\|\alpha \boldsymbol{X}^{-1} \boldsymbol{\Delta} \boldsymbol{x}\right\| & \leq \alpha\left\|\boldsymbol{V}^{-1}\right\|\left\|\boldsymbol{D}^{-1} \boldsymbol{\Delta} \boldsymbol{x}\right\| \\
& \leq \frac{\gamma_{0}^{4} \gamma_{1}^{2} v_{\min }^{2}}{100(n+\nu) \boldsymbol{v}^{T} \boldsymbol{v}} \frac{1}{v_{\min }} \frac{5}{\gamma_{0}^{2} \gamma_{1}} \frac{\boldsymbol{v}^{T} \boldsymbol{v}}{v_{\min }} \\
& \leq 1 / 20, \text { and similarly } \\
\left\|\alpha \boldsymbol{Z}^{-1} \boldsymbol{\Delta} \boldsymbol{z}\right\| & \leq 1 / 20 .
\end{aligned}
$$

These inequalities imply that we have (16) for $\tau:=1 / 20$. Using the above results and Lemmas 3 and 4 in (16), we obtain

$$
\begin{aligned}
\phi\left(\boldsymbol{x}^{k}+\right. & \left.\alpha \boldsymbol{\Delta} \boldsymbol{x}, \boldsymbol{z}^{k}+\alpha \boldsymbol{\Delta} \boldsymbol{z}\right)-\phi\left(\boldsymbol{x}^{k}, \boldsymbol{z}^{k}\right) \\
\leq & -\frac{n+\nu}{\boldsymbol{v}^{T} \boldsymbol{v}}\left\|\boldsymbol{v}-\frac{\boldsymbol{v}^{T} \boldsymbol{v}}{n+\nu} \boldsymbol{V}^{-1} \boldsymbol{e}\right\|^{2} \alpha \\
& +\left(\frac{1}{4} \frac{n+\nu}{\boldsymbol{v}^{T} \boldsymbol{v}}\left\|\boldsymbol{v}-\frac{\boldsymbol{v}^{T} \boldsymbol{v}}{n+\nu} \boldsymbol{V}^{-1} \boldsymbol{e}\right\|^{2}+\frac{10}{19}\left(\left\|\boldsymbol{V}^{-1} \boldsymbol{D}^{-1} \boldsymbol{\Delta} \boldsymbol{x}\right\|^{2}+\left\|\boldsymbol{V}^{-1} \boldsymbol{D} \boldsymbol{\Delta} \boldsymbol{z}\right\|^{2}\right)\right) \alpha^{2} \\
& (49)-\frac{\boldsymbol{v}^{T} \boldsymbol{v}}{n+\nu}\left\|\boldsymbol{V}^{-1} \boldsymbol{e}-\frac{n+\nu}{\boldsymbol{v}^{T} \boldsymbol{v}} \boldsymbol{v}\right\|^{2}\left(1-\frac{\alpha}{4}\right) \alpha+\frac{10}{19}\left\|\boldsymbol{V}^{-2}\right\|\left(\left\|\boldsymbol{D}^{-1} \boldsymbol{\Delta} \boldsymbol{x}\right\|^{2}+\|\boldsymbol{D} \boldsymbol{\Delta} \boldsymbol{z}\|^{2}\right) \alpha^{2} \\
\leq & -\frac{\boldsymbol{v}^{T} \boldsymbol{v}}{n+\nu} \frac{3}{4 v_{\min }^{2}} \frac{399}{400} \alpha+\frac{20}{19} \frac{1}{v_{\min }^{2}} \frac{25}{\gamma_{0}^{4} \gamma_{1}^{2}}\left(\frac{\boldsymbol{v}^{T} \boldsymbol{v}}{v_{\min }}\right)^{2} \alpha^{2} \\
\leq & -\frac{2}{300} \frac{\gamma_{0}^{4} \gamma_{1}^{2}}{(n+\nu)^{2}}+\frac{1}{380} \frac{\gamma_{0}^{4} \gamma_{1}^{2}}{(n+\nu)^{2}} \\
\leq & -\delta .
\end{aligned}
$$

Hence we have (3). The inequality (4) follows from

$$
\begin{aligned}
\left(\boldsymbol{x}^{k}+\alpha \boldsymbol{\Delta} \boldsymbol{x}\right)^{T}\left(\boldsymbol{z}^{k}+\alpha \boldsymbol{\Delta} \boldsymbol{z}\right) & =\left(\boldsymbol{x}^{k}\right)^{T} \boldsymbol{z}^{k}-\left(\left(\boldsymbol{x}^{k}\right)^{T} \boldsymbol{z}^{k}-n\left(\boldsymbol{x}^{k}\right)^{T} \boldsymbol{z}^{k} /(n+\nu)\right) \alpha+\boldsymbol{\Delta} \boldsymbol{x}^{T} \boldsymbol{\Delta} \boldsymbol{z} \alpha^{2} \\
& \geq(1-\alpha)\left(\boldsymbol{x}^{k}\right)^{T} \boldsymbol{z}^{k}+\frac{n\left(\boldsymbol{x}^{k}\right)^{T} \boldsymbol{z}^{k}}{n+\nu} \alpha-\frac{25}{\gamma_{0}^{4} \gamma_{1}^{2}} \frac{\left(\left(\boldsymbol{x}^{k}\right)^{T} \boldsymbol{z}^{k}\right)^{2}}{v_{\min }^{2}} \alpha^{2} \\
& \geq(1-\alpha)\left(\boldsymbol{x}^{k}\right)^{T} \boldsymbol{z}^{k} .
\end{aligned}
$$


4. A pure potential-reduction algorithm. We now consider a potentialreduction algorithm that does not impose the explicit constraint (4) on the step size.

\section{Algorithm II}

Step 1: Choose $\gamma_{0} \in(0,1]$ and a positive constant $\delta$ (which may depend on $n$ and $\nu)$. Set $\left(\boldsymbol{x}^{1}, \boldsymbol{y}^{1}, \boldsymbol{z}^{1}\right):=\gamma_{0} \rho(\boldsymbol{e}, \mathbf{0}, \boldsymbol{e})$. Let $k:=1$.

Step 2: If $\left(\boldsymbol{x}^{k}\right)^{T} \boldsymbol{z}^{k} \leq \epsilon$ then stop.

Step 3: Let $\mu:=\left(\boldsymbol{x}^{k}\right)^{T} \boldsymbol{z}^{k} /(n+\nu)$. Compute the unique solution $(\boldsymbol{\Delta} \boldsymbol{x}, \boldsymbol{\Delta} \boldsymbol{y}, \boldsymbol{\Delta} \boldsymbol{z})$ at $\left(\boldsymbol{x}^{k}, \boldsymbol{y}^{k}, \boldsymbol{z}^{k}\right)$ of the system (2) of equations.

Step 4: Find a step size $\alpha$ such that

$$
\psi\left(\boldsymbol{x}^{k}+\alpha \boldsymbol{\Delta} \boldsymbol{x}, \boldsymbol{y}^{k}+\alpha \boldsymbol{\Delta} \boldsymbol{y}, \boldsymbol{z}^{k}+\alpha \boldsymbol{\Delta} \boldsymbol{z}\right) \leq \psi\left(\boldsymbol{x}^{k}, \boldsymbol{y}^{k}, \boldsymbol{z}^{k}\right)-\delta .
$$

If we cannot find such a step size then stop.

Step 5: Let $\left(\boldsymbol{x}^{k+1}, \boldsymbol{y}^{k+1}, \boldsymbol{z}^{k+1}\right):=\left(\boldsymbol{x}^{k}, \boldsymbol{y}^{k}, \boldsymbol{z}^{k}\right)+\alpha(\boldsymbol{\Delta} \boldsymbol{x}, \boldsymbol{\Delta} \boldsymbol{y}, \boldsymbol{\Delta} \boldsymbol{z})$. Increase $k$ by 1 and go to Step 2 .

The performance of this method is summarized in the following result.

TheOREM 6. Let $L \geq \ln n, \gamma_{0} \in(0,1]$ and $\gamma_{1} \in(0,1)$. Suppose that $\ln \rho=O(L)$, $\ln (1 / \epsilon)=O(L), \nu \geq \sqrt{n}, \sigma:=\gamma_{1}\left(\boldsymbol{x}^{1}\right)^{T} \boldsymbol{z}^{1} /\left\|\left(\boldsymbol{A} \boldsymbol{x}^{1}-\boldsymbol{b}, \boldsymbol{z}^{1}-\boldsymbol{c}\right)\right\|$, and $\delta:=\gamma_{0}^{4} \gamma_{1}^{2} /(300(n+$ $\left.\nu)^{2}\right)$. Then Algorithm II terminates in $O\left(\nu(n+\nu)^{2} L\right)$ iterations. If the algorithm stops in Step 2, we get an approximate solution; otherwise (if it stops in Step 4) there are no optimal solutions $\boldsymbol{x}^{*}$ of $(P)$ and $\left(\boldsymbol{y}^{*}, \boldsymbol{z}^{*}\right)$ of $(D)$ such that $\left\|\left(\boldsymbol{x}^{*}, \boldsymbol{z}^{*}\right)\right\|_{\infty} \leq \rho$.

The proof of this result is like that of Theorem 1. The lemma below shows that it will stop in the required number of iterations.

Lemma 7. Under the assumptions of Theorem $6, \psi\left(\boldsymbol{x}^{1}, \boldsymbol{y}^{1}, \boldsymbol{z}^{1}\right)=O(\nu L)$. If $\psi(\boldsymbol{x}, \boldsymbol{y}, \boldsymbol{z}) \leq \nu \ln \epsilon$ then $\boldsymbol{x}^{T} \boldsymbol{z} \leq \epsilon$.

Proof: It follows from

$$
\begin{aligned}
\psi\left(\boldsymbol{x}^{1}, \boldsymbol{y}^{1}, \boldsymbol{z}^{1}\right) & =\nu \ln \left(n \gamma_{0}^{2} \rho^{2}\right)-\ln \left(1-\gamma_{1}\right), \\
\psi(\boldsymbol{x}, \boldsymbol{y}, \boldsymbol{z}) & =\nu \ln \left(\boldsymbol{x}^{T} \boldsymbol{z}\right)-\sum_{i=1}^{n} \ln \left(\frac{x_{i} z_{i}}{\boldsymbol{x}^{T} \boldsymbol{z} / n}\right)-\ln \left(1-\frac{\sigma\left\|\left(\boldsymbol{A} \boldsymbol{x}-\boldsymbol{b}, \boldsymbol{A}^{T} \boldsymbol{y}+\boldsymbol{z}-\boldsymbol{c}\right)\right\|}{\boldsymbol{x}^{T} \boldsymbol{z}}\right) \\
& \geq \nu \ln \left(\boldsymbol{x}^{T} \boldsymbol{z}\right) .
\end{aligned}
$$

To complete the proof of the theorem, we need to show that if there are optimal solutions $\boldsymbol{x}^{*}$ of $(\mathrm{P})$ and $\left(\boldsymbol{y}^{*}, \boldsymbol{z}^{*}\right)$ of (D) such that $\left\|\left(\boldsymbol{x}^{*}, \boldsymbol{z}^{*}\right)\right\|_{\infty} \leq \rho$, then there exists a step size $\alpha$ which satisfies (20) for $\delta=\gamma_{0}^{4} \gamma_{1}^{2} /\left(300(n+\nu)^{2}\right)$. We use Lemma 5 . Note that (7) holds automatically since $\psi\left(\boldsymbol{x}^{k}, \boldsymbol{y}^{k}, \boldsymbol{z}^{k}\right)$ is finite, using (6) and the definition of $\sigma$. Hence we only need the following result.

Lemma 8. If

$$
\begin{aligned}
\phi\left(\boldsymbol{x}^{k}+\alpha \boldsymbol{\Delta} \boldsymbol{x}, \boldsymbol{z}^{k}+\alpha \boldsymbol{\Delta} \boldsymbol{z}\right) & \leq \phi\left(\boldsymbol{x}^{k}, \boldsymbol{z}^{k}\right)-\delta \\
\left(\boldsymbol{x}^{k}+\alpha \boldsymbol{\Delta} \boldsymbol{x}\right)^{T}\left(\boldsymbol{z}^{k}+\alpha \boldsymbol{\Delta} \boldsymbol{z}\right) & \geq(1-\alpha)\left(\boldsymbol{x}^{k}\right)^{T} \boldsymbol{z}^{k}
\end{aligned}
$$

then

$$
\psi\left(\boldsymbol{x}^{k}+\alpha \boldsymbol{\Delta} \boldsymbol{x}, \boldsymbol{y}^{k}+\alpha \boldsymbol{\Delta} \boldsymbol{y}, \boldsymbol{z}^{k}+\alpha \boldsymbol{\Delta} \boldsymbol{z}\right) \leq \psi\left(\boldsymbol{x}^{k}, \boldsymbol{y}^{k}, \boldsymbol{z}^{k}\right)-\delta
$$

Proof: By (2) we have that

$$
\left(\boldsymbol{A}\left(\boldsymbol{x}^{k}+\alpha \boldsymbol{\Delta} \boldsymbol{x}\right)-\boldsymbol{b}, \boldsymbol{A}^{T}\left(\boldsymbol{y}^{k}+\alpha \boldsymbol{\Delta} \boldsymbol{y}\right)+\left(\boldsymbol{z}^{k}+\alpha \boldsymbol{\Delta} \boldsymbol{z}\right)-\boldsymbol{c}\right)=(1-\alpha)\left(\boldsymbol{A} \boldsymbol{x}^{k}-\boldsymbol{b}, \boldsymbol{A}^{T} \boldsymbol{y}^{k}+\boldsymbol{z}^{k}-\boldsymbol{c}\right) .
$$


Thus we get

$$
\begin{aligned}
& \psi\left(\boldsymbol{x}^{k}+\alpha \boldsymbol{\Delta} \boldsymbol{x}, \boldsymbol{y}^{k}+\alpha \boldsymbol{\Delta} \boldsymbol{y}, \boldsymbol{z}^{k}+\alpha \boldsymbol{\Delta} \boldsymbol{z}\right) \\
& \quad=\phi\left(\boldsymbol{x}^{k}+\alpha \boldsymbol{\Delta} \boldsymbol{x}, \boldsymbol{z}^{k}+\alpha \boldsymbol{\Delta} \boldsymbol{z}\right)-\ln \left(1-\frac{(1-\alpha) \sigma\left\|\left(\boldsymbol{A} \boldsymbol{x}^{k}-\boldsymbol{b}, \boldsymbol{A}^{T} \boldsymbol{y}^{k}+\boldsymbol{z}^{k}-\boldsymbol{c}\right)\right\|}{\left(\boldsymbol{x}^{k}+\alpha \boldsymbol{\Delta}\right)^{T}\left(\boldsymbol{z}^{k}+\alpha \boldsymbol{\Delta} \boldsymbol{z}\right)}\right) \\
& \quad \leq \phi\left(\boldsymbol{x}^{k}, \boldsymbol{z}^{k}\right)-\delta-\ln \left(1-\frac{\sigma\left\|\left(\boldsymbol{A} \boldsymbol{x}^{k}-\boldsymbol{b}, \boldsymbol{A}^{T} \boldsymbol{y}^{k}+\boldsymbol{z}^{k}-\boldsymbol{c}\right)\right\|}{\left(\boldsymbol{x}^{k}\right)^{T} \boldsymbol{z}^{k}}\right) \\
& \quad=\psi\left(\boldsymbol{x}^{k}, \boldsymbol{y}^{k}, \boldsymbol{z}^{k}\right)-\delta .
\end{aligned}
$$

5. An $O(n L)$-iteration variant . Algorithms I and II require $O\left(n^{2.5} L\right)$ iterations. Mizuno [12] proposed an $O(n L)$-iteration variant of the infeasible-interior-point path following algorithm. We can also construct $O(n L)$-iteration variants of Algorithms I and II. In this section, we only show the variant of Algorithm II. Although the $O(n L)$-iteration variant in [12] generates a sequence of infeasible interior points in a neighborhood of the path of centers, our variant does not confine the sequence to such a neighborhood.

\section{Algorithm III}

Step 1: Choose $\gamma_{0}$ and $\lambda$ in $(0,1]$ and positive constants $\delta_{1}$ and $\delta_{2}$. Set $\left(\boldsymbol{x}^{1}, \boldsymbol{y}^{1}, \boldsymbol{z}^{1}\right):=\gamma_{0} \rho(\boldsymbol{e}, \mathbf{0}, \boldsymbol{e})$. Let $k:=1$.

Step 2: If $\left(\boldsymbol{x}^{k}\right)^{T} \boldsymbol{z}^{k} \leq \epsilon$ then stop.

Step 3: If

$$
v_{m i n}^{2}:=\min _{i} x_{i}^{k} z_{i}^{k} \geq \lambda\left(\boldsymbol{x}^{k}\right)^{T} \boldsymbol{z}^{k} / n
$$

then

Step A: Let $\mu:=\left(\boldsymbol{x}^{k}\right)^{T} \boldsymbol{z}^{k} /(n+\nu)$. Compute the unique solution $(\boldsymbol{\Delta} \boldsymbol{x}, \boldsymbol{\Delta} \boldsymbol{y}, \boldsymbol{\Delta} \boldsymbol{z})$ at $\left(\boldsymbol{x}^{k}, \boldsymbol{y}^{k}, \boldsymbol{z}^{k}\right)$ of the system (2) of equations. Find a step size $\alpha$ such that

$$
\psi\left(\boldsymbol{x}^{k}+\alpha \boldsymbol{\Delta} \boldsymbol{x}, \boldsymbol{y}^{k}+\alpha \boldsymbol{\Delta} \boldsymbol{y}, \boldsymbol{z}^{k}+\alpha \boldsymbol{\Delta} \boldsymbol{z}\right) \leq \psi\left(\boldsymbol{x}^{k}, \boldsymbol{y}^{k}, \boldsymbol{z}^{k}\right)-\delta_{1}
$$

If we cannot find such a step size then stop. else

Step B: Compute the unique solution $(\boldsymbol{\Delta} \boldsymbol{x}, \boldsymbol{\Delta} \boldsymbol{y}, \boldsymbol{\Delta} \boldsymbol{z})$ of the system of equations

$$
\left(23\left(\begin{array}{ccc}
\boldsymbol{A} & \mathbf{0} & \mathbf{0} \\
\mathbf{0} & \boldsymbol{A}^{T} & \boldsymbol{I} \\
\boldsymbol{Z}^{k} & \mathbf{0} & \boldsymbol{X}^{k}
\end{array}\right)\left(\begin{array}{c}
\boldsymbol{\Delta} \boldsymbol{x} \\
\boldsymbol{\Delta} \boldsymbol{y} \\
\boldsymbol{\Delta} \boldsymbol{z}
\end{array}\right)=-\left(\begin{array}{c}
\mathbf{0} \\
\mathbf{0} \\
\boldsymbol{X}^{k} \boldsymbol{z}^{k}-\left(\left(\boldsymbol{x}^{k}\right)^{T} \boldsymbol{z}^{k} / n\right) \boldsymbol{e}
\end{array}\right) .\right.
$$

Find a step size $\alpha$ such that

$$
\psi\left(\boldsymbol{x}^{k}+\alpha \boldsymbol{\Delta} \boldsymbol{x}, \boldsymbol{y}^{k}+\alpha \boldsymbol{\Delta} \boldsymbol{y}, \boldsymbol{z}^{k}+\alpha \boldsymbol{\Delta} \boldsymbol{z}\right) \leq \psi\left(\boldsymbol{x}^{k}, \boldsymbol{y}^{k}, \boldsymbol{z}^{k}\right)-\delta_{2} .
$$

Step 4: Let $\left(\boldsymbol{x}^{k+1}, \boldsymbol{y}^{k+1}, \boldsymbol{z}^{k+1}\right):=\left(\boldsymbol{x}^{k}, \boldsymbol{y}^{k}, \boldsymbol{z}^{k}\right)+\alpha(\boldsymbol{\Delta} \boldsymbol{x}, \boldsymbol{\Delta} \boldsymbol{y}, \boldsymbol{\Delta} \boldsymbol{z})$. Increase $k$ by 1 and go to Step 2 .

Note that $(\boldsymbol{\Delta} \boldsymbol{x}, \boldsymbol{\Delta} \boldsymbol{y}, \boldsymbol{\Delta} \boldsymbol{z})$ in Step B is a centering step as in [14]; because of the zeroes in the right-hand side vector in (23), this step maintains the current infeasibilities as well as the "duality gap" $\boldsymbol{x}^{T} \boldsymbol{z}$. 
The performance of this method is summarized in the following result.

TheOREM 9. Let $L \geq \ln n, \gamma_{0} \in(0,1]$ and $\gamma_{1}, \lambda \in(0,1)$. Suppose that $\ln \rho=$ $O(L), \ln (1 / \epsilon)=O(L), \nu \geq n, \sigma:=\gamma_{1}\left(\boldsymbol{x}^{1}\right)^{T} \boldsymbol{z}^{1} /\left\|\left(\boldsymbol{A} \boldsymbol{x}^{1}-\boldsymbol{b}, \boldsymbol{z}^{1}-\boldsymbol{c}\right)\right\|, \delta_{1}:=.001 \lambda^{2} \gamma_{0}^{4} \gamma_{1}^{2}$, and $\delta_{2}:=(1-\lambda)^{2} / 4$. Then Algorithm III terminates in $O(\nu L)$ iterations in Steps 2 or A. If the algorithm stops in Step 2, we get an approximate solution; otherwise (if it stops in Step A) there are no optimal solutions $\boldsymbol{x}^{*}$ of $(P)$ and $\left(\boldsymbol{y}^{*}, \boldsymbol{z}^{*}\right)$ of $(D)$ such that $\left\|\left(\boldsymbol{x}^{*}, \boldsymbol{z}^{*}\right)\right\|_{\infty} \leq \rho$.

Since $\delta_{1}$ and $\delta_{2}$ are constants independent of the input data, the number of iterations is bounded by $O(\nu L)$ (see Lemma 7). As shown in Section 3, we can get an approximate solution if the algorithm stops in Step 2. To complete the proof, we need to show that

(i) if (21) holds, there is a step size $\alpha$ which satisfies (22), or there are no optimal solutions $\boldsymbol{x}^{*}$ of (P) and $\left(\boldsymbol{y}^{*}, \boldsymbol{z}^{*}\right)$ of (D) such that $\left\|\left(\boldsymbol{x}^{*}, \boldsymbol{z}^{*}\right)\right\|_{\infty} \leq \rho$,

(ii) if (21) does not hold, there is a step size $\alpha$ which satisfies (24).

(i) follows from the Lemmas 8 and 11 below. Lemma 10 is used in the proof of Lemma 11. (ii) follows from Lemma 12.

Lemma 10. For any $n$-vector $\boldsymbol{v}>\mathbf{0}$ and any $\nu \geq 0$,

$$
\left\|\boldsymbol{V}^{-1} \boldsymbol{e}-\frac{n+\nu}{\boldsymbol{v}^{T} \boldsymbol{v}} \boldsymbol{v}\right\|^{2} \geq \frac{\nu^{2}}{\boldsymbol{v}^{T} \boldsymbol{v}}
$$

Proof: It follows from

$$
\begin{aligned}
\left\|\boldsymbol{V}^{-1} \boldsymbol{e}-\frac{n+\nu}{\boldsymbol{v}^{T} \boldsymbol{v}} \boldsymbol{v}\right\|^{2} & =\left\|\boldsymbol{V}^{-1} \boldsymbol{e}-\frac{n}{\boldsymbol{v}^{T} \boldsymbol{v}} \boldsymbol{v}\right\|^{2}+\left\|\frac{\nu}{\boldsymbol{v}^{T} \boldsymbol{v}} \boldsymbol{v}\right\|^{2} \\
& \geq \nu^{2} / \boldsymbol{v}^{T} \boldsymbol{v} .
\end{aligned}
$$

Lemma 11. Let $\nu \geq n, \gamma_{0} \in(0,1], \gamma_{1} \in(0,1]$, and $\delta_{1}:=.001 \lambda^{2} \gamma_{0}^{4} \gamma_{1}^{2}$, and suppose (7) and (21) hold. If there exist optimal solutions $\boldsymbol{x}^{*}$ of $(P)$ and $\left(\boldsymbol{y}^{*}, \boldsymbol{z}^{*}\right)$ of $(D)$ such that $\left\|\left(\boldsymbol{x}^{*}, \boldsymbol{z}^{*}\right)\right\|_{\infty} \leq \rho$ then we have (3) and (4) for

$$
\alpha:=\frac{\lambda^{2} \gamma_{0}^{4} \gamma_{1}^{2}}{100(n+\nu)}
$$

at the $k$ th iteration.

Proof: As in the proof of Lemma 5, we have (19) for $\alpha=\lambda^{2} \gamma_{0}^{4} \gamma_{1}^{2} / 100(n+\nu)$. Using Lemma $10, \nu \geq n$, and $v_{m i n}^{2} \geq \lambda \boldsymbol{v}^{T} \boldsymbol{v} / n$, we see that

$$
\begin{aligned}
\phi & \left(\boldsymbol{x}^{k}+\alpha \boldsymbol{\Delta} \boldsymbol{x}, \boldsymbol{z}^{k}+\alpha \boldsymbol{\Delta} \boldsymbol{z}\right)-\phi\left(\boldsymbol{x}^{k}, \boldsymbol{z}^{k}\right) \\
& \leq-\frac{\boldsymbol{v}^{T} \boldsymbol{v}}{n+\nu} \frac{\nu^{2}}{\boldsymbol{v}^{T} \boldsymbol{v}}\left(1-\frac{\alpha}{4}\right) \alpha+\frac{10}{19}\left\|\boldsymbol{V}^{-2}\right\|\left(\left\|\boldsymbol{D}^{-1} \boldsymbol{\Delta} \boldsymbol{x}\right\|^{2}+\|\boldsymbol{D} \boldsymbol{\Delta} \boldsymbol{z}\|^{2}\right) \alpha^{2} \\
& \leq-\frac{\nu^{2}}{n+\nu} \frac{399}{400} \alpha+\frac{20}{19} \frac{1}{v_{\min }^{2}} \frac{25}{\gamma_{0}^{4} \gamma_{1}^{2}}\left(\frac{\boldsymbol{v}^{T} \boldsymbol{v}}{v_{\min }}\right)^{2} \alpha^{2} \\
& \leq-.9 \frac{\nu^{2}}{n+\nu} \alpha+\frac{20}{19} \frac{25}{\gamma_{0}^{4} \gamma_{1}^{2}}\left(\frac{n}{\lambda}\right)^{2} \alpha^{2} \\
& \leq-.009 \lambda^{2} \gamma_{0}^{4} \gamma_{1}^{2} \frac{\nu^{2}}{(n+\nu)^{2}}+\frac{1}{380} \lambda^{2} \gamma_{0}^{4} \gamma_{1}^{2} \frac{n^{2}}{(n+\nu)^{2}} \\
& \leq-\delta_{1} .
\end{aligned}
$$

The inequality (4) follows from the same analysis as in the proof of Lemma 5. 
Lemma 12. (Theorem 6 in Mizuno and Nagasawa [13]) Let $\nu \geq 0$ and $\delta_{2}:=$ $(1-\lambda)^{2} / 4$, and suppose that (21) does not hold. Then we have (24) for

$$
\alpha:=\frac{\lambda_{k}}{1+\sqrt{\lambda_{k} \rho_{k}}}
$$

at the $k$ th iteration, where $\lambda_{k}:=\min _{i} \frac{x_{i}^{k} z_{i}^{k}}{\left(\boldsymbol{x}^{k}\right)^{T} \boldsymbol{z}^{k} / n}$ and $\rho_{k}:=\sum_{i=1}^{n}\left(\frac{\left(\boldsymbol{x}^{k}\right)^{T} \boldsymbol{z}^{k} / n}{x_{i}^{k} z_{i}^{k}}-1\right)$. The result above is proved in [13] for the potential function $\phi$ with $\nu=\sqrt{n}$, but it is valid for the potential function $\psi$ with any $\nu \geq 0$ since the duality gap and infeasibility do not change in Step B.

6. Concluding remarks. In this final section we contrast Algorithms I and II, discuss the results obtained, and briefly consider other possible primal-dual potential functions for the infeasible case. We also describe an extension to monotone linear complementarity problems.

\section{(A) Getting information on infeasibility.}

We note that $\gamma_{0}$ and $\rho$ appear in the algorithms only through their product and the dependence of $\delta$ on $\gamma_{0}$. Suppose that we start Algorithm I, II or III with $\left(\boldsymbol{x}^{1}, \boldsymbol{y}^{1}, \boldsymbol{z}^{1}\right)=$ $\rho_{0}(\boldsymbol{e}, \mathbf{0}, \boldsymbol{e})$ for some $\rho_{0}>0$, and that we perform at each iteration a line search to achieve the largest decrease in $\phi$ subject to satisfying (4) (largest decrease in $\psi$ ).

If there are optimal solutions $\boldsymbol{x}^{*}$ of (P) and $\left(\boldsymbol{y}^{*}, \boldsymbol{z}^{*}\right)$ of (D) such that $\left\|\left(\boldsymbol{x}^{*}, \boldsymbol{z}^{*}\right)\right\|_{\infty} \leq$ $\rho_{0} / \gamma_{0}$ for some $\gamma_{0} \in(0,1]$, it follows from the inequality (9) in the proof of Lemma 4 that

$$
\theta^{k} \rho_{0}\left\|\left(\boldsymbol{x}^{k}, \boldsymbol{z}^{k}\right)\right\|_{1} \leq \frac{2\left(\boldsymbol{x}^{k}\right)^{T} \boldsymbol{z}^{k}}{\gamma_{0} \gamma_{1}}
$$

Hence if this inequality is violated, then we can conclude that there are no optimal solutions $\boldsymbol{x}^{*}$ of (P) and $\left(\boldsymbol{y}^{*}, \boldsymbol{z}^{*}\right)$ of (D) such that $\left\|\left(\boldsymbol{x}^{*}, \boldsymbol{z}^{*}\right)\right\|_{\infty} \leq \rho_{0} / \gamma_{0}$. Here $\gamma_{0}$ can be varied during one run of the algorithm.

\section{(B) Comparison between Algorithms I and II.}

In Algorithm I, we put an explicit bound on $\alpha$ via (4), in order to ensure that

$$
\left(\boldsymbol{x}^{k}\right)^{T} \boldsymbol{z}^{k} \geq \frac{\left\|\left(\boldsymbol{A} \boldsymbol{x}^{k}-\boldsymbol{b}, \boldsymbol{A}^{T} \boldsymbol{y}^{k}+\boldsymbol{z}^{k}-\boldsymbol{c}\right)\right\|}{\left\|\left(\boldsymbol{A} \boldsymbol{x}^{1}-\boldsymbol{b}, \boldsymbol{A}^{T} \boldsymbol{y}^{1}+\boldsymbol{z}^{1}-\boldsymbol{c}\right)\right\|}\left(\boldsymbol{x}^{1}\right)^{T} \boldsymbol{z}^{1}
$$

for all $k$. Inequalities of this kind were first used by Kojima, Megiddo and Mizuno [3]. In fact, (4) can be relaxed as long as (25) holds at each iteration; if (4) held strictly at some previous iteration, (25) may hold even if (4) does not. Algorithms II and III dispense with this explicit constraint by adding a barrier term to $\phi$. If $\sigma$ is as in Theorem 6 , then $\psi$ is only finite if

$$
\left(\boldsymbol{x}^{k}\right)^{T} \boldsymbol{z}^{k} \geq \gamma_{1} \frac{\left\|\left(\boldsymbol{A} \boldsymbol{x}^{k}-\boldsymbol{b}, \boldsymbol{A}^{T} \boldsymbol{y}^{k}+\boldsymbol{z}^{k}-\boldsymbol{c}\right)\right\|}{\left\|\left(\boldsymbol{A} \boldsymbol{x}^{1}-\boldsymbol{b}, \boldsymbol{A}^{T} \boldsymbol{y}^{1}+\boldsymbol{z}^{1}-\boldsymbol{c}\right)\right\|}\left(\boldsymbol{x}^{1}\right)^{T} \boldsymbol{z}^{1} .
$$

For $\gamma_{1}<1$, this is a weaker condition than (25), but the complexity bounds include a factor $\gamma_{1}^{-2}$. 
In contrast, the homogeneous self-dual infeasible-interior-point algorithm of Ye, Todd and Mizuno [21] maintains

$$
\boldsymbol{c}^{T} \boldsymbol{x}^{k}-\boldsymbol{b}^{T} \boldsymbol{y}^{k} \leq \frac{\left\|\left(\boldsymbol{A} \boldsymbol{x}^{k}-\boldsymbol{b} \tau^{k}\right)\right\|}{\left\|\left(\boldsymbol{A} \boldsymbol{x}^{1}-\boldsymbol{b}\right)\right\|}\left(\boldsymbol{c}^{T} \boldsymbol{x}^{1}-\boldsymbol{b}^{T} \boldsymbol{y}^{1}+1\right)
$$

in the present notation. Here, $\tau^{k}$ is the value of the homogenizing variable at the $k$ th iteration; $\tau^{1}=1$. Hence in [21] the "duality gap" decreases faster than the infeasibility, whereas in the current paper as well as $[3,22,12]$ the "total complementarity" decreases at most as fast as the infeasibility. (Note that, with infeasible iterates, $\boldsymbol{c}^{T} \boldsymbol{x}^{k}-\boldsymbol{b}^{T} \boldsymbol{y}^{k}$ may not equal $\left(\boldsymbol{x}^{k}\right)^{T} \boldsymbol{z}^{k}$, and may even be negative.)

\section{(C) Complexity Bounds.}

Our bound on the number of iterations for Algorithms I and II is $O\left(n^{2.5} L\right)$ (when $\nu=\sqrt{n}$ ), while Zhang [22] and Mizuno [12] obtain $O\left(n^{2} L\right)$ (Mizuno has a variant with $O(n L))$ and Potra [17] achieves $O\left(n^{1.5} L\right)$; in contrast, feasible-interior-point algorithms typically have bounds of $O\left(n^{.5} L\right)$ iterations [5, 6, 15, 16]. Let us examine why the complexity is so much larger in our case, and why it decreases for Algorithm III.

Since the analysis for Algorithm II is based on that for Algorithm I, we consider only the latter. We also assume $\nu \leq 3 n$. Using the arguments of Lemmas 2 and 5 , we have for any $0<\alpha<1$ satisfying

$$
\left\|\alpha\left(\boldsymbol{X}^{k}\right)^{-1} \boldsymbol{\Delta} \boldsymbol{x}\right\| \leq \tau,\left\|\alpha\left(\boldsymbol{Z}^{k}\right)^{-1} \boldsymbol{\Delta} \boldsymbol{z}\right\| \leq \tau
$$

for some $\tau \in(0,1)$,

$$
\begin{aligned}
\Delta \phi:= & \phi\left(\boldsymbol{x}^{k}+\alpha \boldsymbol{\Delta} \boldsymbol{x}, \boldsymbol{z}^{k}+\alpha \boldsymbol{\Delta} \boldsymbol{z}\right)-\phi\left(\boldsymbol{x}^{k}, \boldsymbol{z}^{k}\right) \\
\leq & -\frac{\boldsymbol{v}^{T} \boldsymbol{v}}{n+\nu}\left\|\boldsymbol{V}^{-1} \boldsymbol{e}-\frac{n+\nu}{\boldsymbol{v}^{T} \boldsymbol{v}} \boldsymbol{v}\right\|^{2} \frac{3}{4} \alpha \\
& +\frac{1}{2(1-\tau)}\left\|\boldsymbol{V}^{-2}\right\|\left(\left\|\boldsymbol{D}^{-1} \boldsymbol{\Delta} \boldsymbol{x}\right\|^{2}+\|\boldsymbol{D} \boldsymbol{\Delta} \boldsymbol{z}\|^{2}\right) \alpha^{2}
\end{aligned}
$$

(cf. (19)). In our analysis, we bounded the second-order term above using Lemma 4; using also Lemma 3 to bound the first-order term, we get

$$
\begin{aligned}
\Delta \phi \leq & -\frac{3}{4}\left(\frac{\sqrt{3}}{2}\right)^{2} \frac{\boldsymbol{v}^{T} \boldsymbol{v}}{n+\nu} v_{\min }^{-2} \alpha \\
& +\frac{1}{2(1-\tau)} v_{\min }^{-2} \frac{50}{\gamma_{0}^{4} \gamma_{1}^{2}} \frac{\left(\boldsymbol{v}^{T} \boldsymbol{v}\right)^{2}}{v_{m i n}^{2}} \alpha^{2}
\end{aligned}
$$

The linear term in (28) would allow a constant decrease in $\phi$ by choosing a constant $\alpha$, but unfortunately the quadratic term is much too large. Indeed, the right-hand side of (28) is minimized by

$$
\alpha=\frac{9(1-\tau)}{800} \frac{\gamma_{0}^{4} \gamma_{1}^{2} v_{\min }^{2} n}{\boldsymbol{v}^{T} \boldsymbol{v}} \frac{1}{n(n+\nu)} .
$$

Notice that $n v_{\text {min }}^{2}=\boldsymbol{v}^{T} \boldsymbol{v}$ for $\boldsymbol{v}$ a multiple of $\boldsymbol{e}$, i.e., when $\left(\boldsymbol{x}^{k}, \boldsymbol{y}^{k}, \boldsymbol{z}^{k}\right)$ is on the central path, and then $\alpha=O\left(n^{-2}\right)$ and hence $\Delta \phi=O\left(n^{-2}\right)$. Our choice for $\alpha$ in Lemma 5 approximates this "optimal" value. 
Now let us see how this analysis changes when the iterate is feasible. Note that

$$
\begin{aligned}
\left\|\boldsymbol{D}^{-1} \boldsymbol{\Delta} \boldsymbol{x}\right\|^{2}+\|\boldsymbol{D} \boldsymbol{\Delta} \boldsymbol{z}\|^{2} & =\left\|\boldsymbol{D}^{-1} \boldsymbol{\Delta} \boldsymbol{x}+\boldsymbol{D} \boldsymbol{\Delta} \boldsymbol{z}\right\|^{2}-2 \boldsymbol{\Delta} \boldsymbol{x}^{T} \boldsymbol{\Delta} \boldsymbol{z} \\
& =\left(\frac{\boldsymbol{v}^{T} \boldsymbol{v}}{n+\nu}\right)^{2}\left\|\boldsymbol{V}^{-1} e-\frac{n+\nu}{\boldsymbol{v}^{T} \boldsymbol{v}} \boldsymbol{v}\right\|^{2}-2 \boldsymbol{\Delta} \boldsymbol{x}^{T} \boldsymbol{\Delta} \boldsymbol{z}
\end{aligned}
$$

The last equality follows from the final equation of (2). In the feasible case, $\Delta x^{T} \boldsymbol{\Delta} \boldsymbol{z}$ is zero, and, for $\left\|\boldsymbol{V}^{-1} \boldsymbol{e}-\frac{n+\nu}{\boldsymbol{v}^{\mathrm{T}} \boldsymbol{v}} \boldsymbol{v}\right\|=O\left(v_{\text {min }}^{-1}\right)$, the resulting second-order term is smaller than in (28) by a factor of about $n^{2}$. We can thus choose a much larger value for $\alpha$; indeed,

$$
\alpha:=\frac{\tau(n+\nu) v_{\min }}{\boldsymbol{v}^{T} \boldsymbol{v}\left\|\boldsymbol{V}^{-1} \boldsymbol{e}-\frac{n+\nu}{\boldsymbol{v}^{T} \boldsymbol{v}} \boldsymbol{v}\right\|}
$$

for $\tau:=\sqrt{3} / 9$ satisfies all our requirements and yields $\Delta \phi \leq-1 / 16$.

The equations in (29) show that $\left\|\boldsymbol{D}^{-1} \boldsymbol{\Delta} \boldsymbol{x}\right\|^{2}+\|\boldsymbol{D} \boldsymbol{\Delta} \boldsymbol{z}\|^{2}$ is much larger than $\left\|D^{-1} \boldsymbol{\Delta} \boldsymbol{x}+\boldsymbol{D} \boldsymbol{\Delta} \boldsymbol{z}\right\|^{2}$ when $\boldsymbol{\Delta} \boldsymbol{x}^{T} \boldsymbol{\Delta} \boldsymbol{z}$ is large and negative. (It cannot be large and positive by (18), but this doesn't help us; (17) provides no lower bound.) But from (11) and (15), we obtain

$$
\begin{aligned}
\boldsymbol{\Delta} \boldsymbol{x}^{T} \boldsymbol{\Delta} \boldsymbol{z}= & \left(\boldsymbol{D}^{-1} \boldsymbol{\Delta} \boldsymbol{x}\right)^{T}(\boldsymbol{D} \boldsymbol{\Delta} \boldsymbol{z}) \\
= & -\left(\theta^{k}\right)^{2}\left(\boldsymbol{x}^{1}-\boldsymbol{x}^{*}\right)^{T} \boldsymbol{D}^{-1} \boldsymbol{Q} \boldsymbol{D}^{-1}\left(\boldsymbol{x}^{1}-\boldsymbol{x}^{*}\right)-\left(\theta^{k}\right)^{2}\left(\boldsymbol{z}^{1}-\boldsymbol{z}^{*}\right)^{T} \boldsymbol{D}(\boldsymbol{I}-\boldsymbol{Q}) \boldsymbol{D}\left(\boldsymbol{z}^{1}-\boldsymbol{z}^{*}\right) \\
& +\theta^{k}\left(\boldsymbol{x}^{1}-\boldsymbol{x}^{*}\right)^{T} \boldsymbol{D}^{-1} \boldsymbol{Q}\left(\boldsymbol{v}-\mu \boldsymbol{V}^{-1} e\right)+\theta^{k}\left(\boldsymbol{z}^{1}-\boldsymbol{z}^{*}\right)^{T} \boldsymbol{D}(\boldsymbol{I}-\boldsymbol{Q})\left(\boldsymbol{v}-\mu \boldsymbol{V}^{-1} e\right) .
\end{aligned}
$$

The first two terms are negative, while the last two are of indeterminate sign. It is not hard to see that

$$
\begin{aligned}
\left(\theta^{k}\right)^{2}\left(\boldsymbol{x}^{1}-\boldsymbol{x}^{*}\right)^{T} \boldsymbol{D}^{-1} \boldsymbol{Q} \boldsymbol{D}^{-1}\left(\boldsymbol{x}^{1}-\boldsymbol{x}^{*}\right) & =\left(\boldsymbol{x}^{k}-\boldsymbol{x}^{*}\right)^{T} \boldsymbol{D}^{-1} \boldsymbol{Q} \boldsymbol{D}^{-1}\left(\boldsymbol{x}^{k}-\boldsymbol{x}^{*}\right) \\
& =\left\|\boldsymbol{Q} \boldsymbol{D}^{-1}\left(\boldsymbol{x}^{k}-\boldsymbol{x}^{*}\right)\right\|^{2},
\end{aligned}
$$

and this can be seen to be the square of the distance from $D^{-1} x^{k}$ to the affine set $\{\boldsymbol{x}: \boldsymbol{A D} \boldsymbol{x}=\boldsymbol{b}\}$. Similarly,

$$
\begin{aligned}
\left(\theta^{k}\right)^{2}\left(\boldsymbol{z}^{1}-\boldsymbol{z}^{*}\right)^{T} \boldsymbol{D}(\boldsymbol{I}-\boldsymbol{Q}) \boldsymbol{D}\left(\boldsymbol{z}^{1}-\boldsymbol{z}^{*}\right) & =\left(\boldsymbol{z}^{k}-\boldsymbol{z}^{*}\right)^{T} \boldsymbol{D}(\boldsymbol{I}-\boldsymbol{Q}) \boldsymbol{D}\left(\boldsymbol{z}^{k}-\boldsymbol{z}^{*}\right) \\
& =\left\|(\boldsymbol{I}-\boldsymbol{Q}) \boldsymbol{D}\left(\boldsymbol{z}^{k}-\boldsymbol{z}^{*}\right)\right\|^{2}
\end{aligned}
$$

is the square of the distance from $\boldsymbol{D} \boldsymbol{z}^{k}$ to the affine set $\left\{\boldsymbol{z}: \boldsymbol{D} \boldsymbol{A}^{T} \boldsymbol{y}+\boldsymbol{z}=\boldsymbol{D} \boldsymbol{c}\right.$ for some $\left.\boldsymbol{y}\right\}$. The last two terms are bounded by $\left\|\boldsymbol{Q} \boldsymbol{D}^{-1}\left(\boldsymbol{x}^{k}-\boldsymbol{x}^{*}\right)\right\|\left\|\boldsymbol{v}-\mu \boldsymbol{V}^{-1} e\right\|$ and $\|(\boldsymbol{I}-\boldsymbol{Q}) \boldsymbol{D}\left(\boldsymbol{z}^{k}-\right.$ $\left.\boldsymbol{z}^{*}\right)\|\| \boldsymbol{v}-\mu \boldsymbol{V}^{-1} e \|$. If $\boldsymbol{v}$ is a multiple of $\boldsymbol{e}\left(\left(\boldsymbol{x}^{k}, \boldsymbol{y}^{k}, \boldsymbol{z}^{k}\right)\right.$ is on the central path), then $\left\|\boldsymbol{v}-\mu \boldsymbol{V}^{-1} e\right\|$ is bounded by $\sqrt{\left(\boldsymbol{x}^{k}\right)^{T} \boldsymbol{z}^{k}}$. Hence if the infeasibility is large compared to the duality gap, $\boldsymbol{\Delta} \boldsymbol{x}^{T} \boldsymbol{\Delta} \boldsymbol{z}$ will be large and negative, the second order term in (27) will be large, and only a small decrease in $\phi$ can be guaranteed. This also explains why we need to balance carefully the infeasibility and the duality gap, as in (25)-(26).

We remark that, if the 2-norm of $\left(\boldsymbol{x}^{k}, \boldsymbol{z}^{k}\right)$ is much smaller than its 1-norm (as when, for instance, it is close to a multiple of $(\boldsymbol{e}, \boldsymbol{e})$ ), then the first inequality in (13), and hence the bound (14) on $\left\|\boldsymbol{D}^{-1} \boldsymbol{\Delta} \boldsymbol{x}\right\|$ and similarly that on $\|\boldsymbol{D} \boldsymbol{\Delta} \boldsymbol{z}\|$, could be improved by a factor close to $\sqrt{n}$. Then $\alpha$ could be chosen to give a reduction in $\phi$ of order $n^{-1}$ rather than $n^{-2}$.

Finally, Lemma 10 allows us to obtain a better bound on the first term in (27) when the current iterate is approximately centered, and in this case the second-order 
term is smaller and thus a greater decrease in $\phi$ (and $\psi$ ) can be achieved by choosing a larger value for $\alpha$. This is the basis for Step A in Algorithm III. Lemma 12 proves that a constant decrease in $\phi$ (and $\psi$ ) can also be achieved when the current iterate is far from centered, by using a simple centering step.

\section{(D) Some other potential functions.}

There are two other primal-dual potential functions that could be used in the infeasible case. The first is

$$
\psi^{\prime}(\boldsymbol{x}, \boldsymbol{y}, \boldsymbol{z}):=(n+\nu) \ln \left(\boldsymbol{x}^{T} \boldsymbol{z}+\kappa\left\|\left(\boldsymbol{A} \boldsymbol{x}-\boldsymbol{b}, \boldsymbol{A}^{T} \boldsymbol{y}+\boldsymbol{z}-\boldsymbol{c}\right)\right\|\right)-\sum_{i=1}^{n} \ln \left(x_{i} z_{i}\right)-n \ln n,
$$

and was suggested for a pure potential-reduction method by Kojima, Noma, and Yoshise [7] in the context of the monotone complementarity problem. The second is

$\psi^{\prime \prime}(\boldsymbol{x}, \boldsymbol{y}, \boldsymbol{z}):=(n+\nu) \ln \left(\max \left\{\boldsymbol{x}^{T} \boldsymbol{z}, \kappa\left\|\left(\boldsymbol{A} \boldsymbol{x}-\boldsymbol{b}, \boldsymbol{A}^{T} \boldsymbol{y}+\boldsymbol{z}-\boldsymbol{c}\right)\right\|\right\}\right)-\sum_{i=1}^{n} \ln \left(x_{i} z_{i}\right)-n \ln n$,

Both can be reduced by an amount sufficient to establish a polynomial time bound if we add a restriction like (4) on the step size, so that (25) holds for all $k$. However, in this case there seems to be no reason to choose these more complicated functions over the simpler $\phi$. If we relax the constraint, Kojima, Noma, and Yoshise [7] show that $\psi^{\prime}$ can always be reduced by some amount, but provide no bound (indeed, it seems hard to do so, even in the case of linear programming). Similar difficulties arise with $\psi^{\prime \prime}$. It seems to be very hard to obtain a guaranteed decrease in such a potential function when the duality gap $\boldsymbol{x}^{T} \boldsymbol{z}$ is much smaller than the infeasibility $\left\|\left(\boldsymbol{A} \boldsymbol{x}-\boldsymbol{b}, \boldsymbol{A}^{T} \boldsymbol{y}+\boldsymbol{z}-\boldsymbol{c}\right)\right\|$. We also mention a modified primal-dual potential function given by Kaliski and Ye [1] for a monotone linear complementarity problem with a restriction that some prescribed variables are zero. Their algorithm with the use of the modified potential function solves a combined Phase I-Phase II primal-dual linear program in $O(n L)$ iterations.

(E) Extension to monotone linear complementarity problems.

Consider a linear complementarity problem with a positive semi-definite matrix $\boldsymbol{M}$ and a vector $\boldsymbol{q}$ : Find a pair $(\boldsymbol{x}, \boldsymbol{z}) \geq \mathbf{0}$ such that $\boldsymbol{z}=\boldsymbol{M} \boldsymbol{x}+\boldsymbol{q}$ and $\boldsymbol{x}^{T} \boldsymbol{z}=\mathbf{0}$. We can easily adapt Algorithms I, II and III to the problem. Major changes are:

- Eliminate $\boldsymbol{y}^{1}, \boldsymbol{y}^{k}, \boldsymbol{y}^{k+1}$ and $\boldsymbol{\Delta} \boldsymbol{y}$.

- Replace the system (2) of equations by

$$
\left(\begin{array}{cc}
-\boldsymbol{M} & \boldsymbol{I} \\
\boldsymbol{Z}^{k} & \boldsymbol{X}^{k}
\end{array}\right)\left(\begin{array}{c}
\Delta \boldsymbol{x} \\
\Delta \boldsymbol{z}
\end{array}\right)=-\left(\begin{array}{c}
\boldsymbol{z}^{k}-\boldsymbol{M} \boldsymbol{x}^{k}-\boldsymbol{q} \\
\boldsymbol{X}^{k} \boldsymbol{z}^{k}-\mu \boldsymbol{e}
\end{array}\right)
$$

- Replace the system (23) of equations by

$$
\left(\begin{array}{cc}
-\boldsymbol{M} & \boldsymbol{I} \\
\boldsymbol{Z}^{k} & \boldsymbol{X}^{k}
\end{array}\right)\left(\begin{array}{c}
\Delta \boldsymbol{x} \\
\boldsymbol{\Delta} \boldsymbol{z}
\end{array}\right)=-\left(\begin{array}{c}
\mathbf{0} \\
\boldsymbol{X}^{k} \boldsymbol{z}^{k}-\left(\left(\boldsymbol{x}^{k}\right)^{T} \boldsymbol{z}^{k} / n\right) \boldsymbol{e}
\end{array}\right)
$$

- Replace the potential function $\psi$ by

$$
\begin{aligned}
\psi(\boldsymbol{x}, \boldsymbol{z}):= & (n+\nu+1) \ln \left(\boldsymbol{x}^{T} \boldsymbol{z}\right)-\sum_{i=1}^{n} \ln \left(x_{i} z_{i}\right)-n \ln n \\
& -\ln \left(\boldsymbol{x}^{T} \boldsymbol{z}-\sigma\|\boldsymbol{z}-\boldsymbol{M} \boldsymbol{x}-\boldsymbol{q}\|\right) .
\end{aligned}
$$


Then we have results similar to Theorems 1,6 , and 9 , whose proofs are basically the same as in the linear programming case except for Lemma 4 . In the proof of Lemma 4 , we have

$$
\begin{gathered}
\left(\theta^{k} \boldsymbol{x}^{1}+\left(1-\theta^{k}\right) \boldsymbol{x}^{*}-\boldsymbol{x}^{k}\right)^{T}\left(\theta^{k} \boldsymbol{z}^{1}+\left(1-\theta^{k}\right) \boldsymbol{z}^{*}-\boldsymbol{z}^{k}\right) \geq 0 \\
\left(\begin{array}{cc}
-\boldsymbol{M} & \boldsymbol{I} \\
\boldsymbol{Z}^{k} & \boldsymbol{X}^{k}
\end{array}\right)\left(\begin{array}{c}
\boldsymbol{\Delta} \boldsymbol{x}+\theta^{k}\left(\boldsymbol{x}^{1}-\boldsymbol{x}^{*}\right) \\
\boldsymbol{\Delta} \boldsymbol{z}+\theta^{k}\left(\boldsymbol{z}^{1}-\boldsymbol{z}^{*}\right)
\end{array}\right)=-\left(\begin{array}{c}
\mathbf{0} \\
\boldsymbol{X}^{k} \boldsymbol{z}^{k}-\mu \boldsymbol{e}-\theta^{k} \boldsymbol{Z}^{k}\left(\boldsymbol{x}^{1}-\boldsymbol{x}^{*}\right)-\theta^{k} \boldsymbol{X}^{k}\left(\boldsymbol{z}^{1}-\boldsymbol{z}^{*}\right)
\end{array}\right),
\end{gathered}
$$

instead of (8) and (10), respectively. It is well known in interior-point methods for LCP (and easy to show) that for any $n$-dimensional vector $\boldsymbol{p}$, the solution of the system

$$
\left(\begin{array}{cc}
-M & I \\
Z^{k} & X^{k}
\end{array}\right)\left(\begin{array}{c}
\Delta x^{\prime} \\
\Delta z^{\prime}
\end{array}\right)=\left(\begin{array}{c}
0 \\
p
\end{array}\right)
$$

satisfies

$$
\begin{aligned}
\left\|\boldsymbol{D}^{-1} \boldsymbol{\Delta} \boldsymbol{x}^{\prime}\right\| & =\left\|\boldsymbol{D}\left(\boldsymbol{\Delta} \boldsymbol{z}^{\prime}-\left(\boldsymbol{X}^{k}\right)^{-1} \boldsymbol{p}\right)\right\| \leq\left\|\left(\boldsymbol{X}^{k} \boldsymbol{Z}^{k}\right)^{-1 / 2} \boldsymbol{p}\right\|, \\
\left\|\boldsymbol{D} \boldsymbol{\Delta} \boldsymbol{z}^{\prime}\right\| & =\left\|\boldsymbol{D}^{-1}\left(\boldsymbol{\Delta} \boldsymbol{x}^{\prime}-\left(\boldsymbol{Z}^{k}\right)^{-1} \boldsymbol{p}\right)\right\| \leq\left\|\left(\boldsymbol{X}^{k} \boldsymbol{Z}^{k}\right)^{-1 / 2} \boldsymbol{p}\right\|,
\end{aligned}
$$

where $\boldsymbol{D}=\left(\boldsymbol{X}^{k}\right)^{1 / 2}\left(\boldsymbol{Z}^{k}\right)^{-1 / 2}$. Let $\left(\boldsymbol{\Delta} \boldsymbol{x}_{1}^{\prime}, \boldsymbol{\Delta} \boldsymbol{z}_{1}^{\prime}\right),\left(\boldsymbol{\Delta} \boldsymbol{x}_{2}^{\prime}, \boldsymbol{\Delta} \boldsymbol{z}_{2}^{\prime}\right)$, and $\left(\boldsymbol{\Delta} \boldsymbol{x}_{3}^{\prime}, \boldsymbol{\Delta} \boldsymbol{z}_{3}^{\prime}\right)$ be the solution of the system above when $\boldsymbol{p}$ is $-\left(\boldsymbol{X}^{k} \boldsymbol{z}^{k}-\mu \boldsymbol{e}\right), \theta^{k} \boldsymbol{Z}^{k}\left(\boldsymbol{x}^{1}-\boldsymbol{x}^{*}\right)$, and $\theta^{k} \boldsymbol{X}^{k}\left(\boldsymbol{z}^{1}-\boldsymbol{z}^{*}\right)$ respectively. Then we have

$$
\begin{aligned}
\left\|D^{-1} \Delta x\right\| & =\left\|D^{-1}\left(\Delta x_{1}^{\prime}+\Delta x_{2}^{\prime}+\Delta x_{3}^{\prime}-\theta^{k}\left(\boldsymbol{x}^{1}-x^{*}\right)\right)\right\| \\
& \leq\left\|\boldsymbol{D}^{-1}\left(\Delta x_{1}^{\prime}+\Delta x_{3}^{\prime}\right)\right\|+\left\|\boldsymbol{D} \Delta \boldsymbol{z}_{2}^{\prime}\right\| \\
& \leq \theta^{k}\left\|\boldsymbol{D}^{-1}\left(\boldsymbol{x}^{1}-\boldsymbol{x}^{*}\right)\right\|+\theta^{k}\left\|\boldsymbol{D}\left(\boldsymbol{z}^{1}-\boldsymbol{z}^{*}\right)\right\|+\left\|\left(\boldsymbol{X}^{k} \boldsymbol{Z}^{k}\right)^{-1 / 2}\left(\boldsymbol{X}^{k} \boldsymbol{z}^{k}-\mu \boldsymbol{e}\right)\right\|
\end{aligned}
$$

and similarly

$$
\|\boldsymbol{D} \boldsymbol{\Delta} \boldsymbol{z}\| \leq \theta^{k}\left\|\boldsymbol{D}^{-1}\left(\boldsymbol{x}^{1}-\boldsymbol{x}^{*}\right)\right\|+\theta^{k}\left\|\boldsymbol{D}\left(\boldsymbol{z}^{1}-\boldsymbol{z}^{*}\right)\right\|+\left\|\left(\boldsymbol{X}^{k} \boldsymbol{Z}^{k}\right)^{-1 / 2}\left(\boldsymbol{X}^{k} \boldsymbol{z}^{k}-\mu \boldsymbol{e}\right)\right\| .
$$

Thus we can prove the lemma following the same arguments as before.

\section{REFERENCES}

[1] J. A. KALISKi AND Y. YE, An extension of the potential reduction algorithm for solving the linear complentary problem with priority goals, Linear Algebra and its Applications, 193 (1993), pp. $35-50$.

[2] N. K. KARMARKAR, A new polynomial-time algorithm for linear programming, Combinatorica, 4 (1984), pp. 373-395.

[3] M. Kojima, N. Megiddo, and S. Mizuno, A primal-dual infeasible-interior-point algorithm for linear programming, Mathematical Programming, 61 (1993), pp. 263-280.

[4] M. Kojima, S. Mizuno, And A. Yoshise, A primal-dual interior point algorithm for linear programming, in Progress in Mathematical Programming : Interior Point and Related Methods, N. Megiddo, ed., Springer Verlag, New York, 1989, pp. 29-47.

[5] — A polynomial-time algorithm for a class of linear complementarity problems, Mathematical Programming, 44 (1989), pp. 1-26.

[6] - An $O(\sqrt{n} L)$ iteration potential reduction algorithm for linear complementarity problems, Mathematical Programming, 50 (1991), pp. 331-342.

[7] M. KojIma, T. Noma, AND A. Yoshise, Global convergence and detecting infeasibility in interior-point algorithms, Research Reports on Information Sciences, Ser. B : Operations Research B-257, Dept. of Information Sciences, Tokyo Institute of Technology, OhOkayama, Meguro-ku, Tokyo 152, Japan, September 1992. 
[8] I. J. Lustig, Feasibility issues in a primal-dual interior point method for linear programming, Mathematical Programming, 49 (1990/91), pp. 145-162.

[9] I. J. Lustig, R. E. Marsten, And D. F. Shanno, Computational experience with a primaldual interior point method for linear programming, Linear Algebra and Its Applications, 152 (1991), pp. 191-222.

[10] R. E. Marsten, R. Subramaniam, M. J. Saltzman, I. J. Lustig, and D. F. Shanno, Interior point methods for linear programming : Just call Newton, Lagrange, and Fiacco and McCormick!, Interfaces, 20 (1990), pp. 105-116.

[11] N. Megiddo, Pathways to the optimal set in linear programming, in Progress in Mathematical Programming : Interior Point and Related Methods, N. Megiddo, ed., Springer Verlag, New York, 1989, pp. 131-158. Identical version in : Proceedings of the 6th Mathematical Programming Symposium of Japan, Nagoya, Japan, pages 1-35, 1986.

[12] S. Mizuno, Polynomiality of the Kojima-Megiddo-Mizuno infeasible interior point algorithm for linear programming, Technical Report 1006, School of Operations Research and Industrial Engineering, Cornell University, Ithaca, NY 14853-3801, USA, May 1992.

[13] S. Mizuno And A. Nagasawa, A primal-dual affine scaling potential reduction algorithm for linear programming, Mathematical Programming, 62 (1993), pp. 119-131.

[14] S. Mizuno, M. J. TodD, AND Y. YE, On adaptive step primal-dual interior-point algorithms for linear programming, Mathematics of Operations Research, 18 (1993), pp. 964-981.

[15] R. D. C. MONTEIRo AND I. AdLeR, Interior path following primal-dual algorithms : Part I : Linear programming, Mathematical Programming, 44 (1989), pp. 27-41.

[16] - Interior path following primal-dual algorithms : Part II : Convex quadratic programming, Mathematical Programming, 44 (1989), pp. 43-66.

[17] F. A. PotrA, An infeasible interior-point predictor-corrector algorithm for linear programming, Reports on Computational Mathematics 26, Dept. of Mathematics, The University of Iowa, Iowa City, IA 52242, USA, June 1992.

[18] K. Tanabe, Centered Newton method for mathematical programming, in System Modelling and Optimization : Proceedings of the 13th IFIP Conference, Tokyo, Japan, Aug./Sept. 1987, M. Iri and K. Yajima, eds., vol. 113 of Lecture Notes in Control and Information Sciences, Springer Verlag, Berlin, West-Germany, 1988, pp. 197-206.

[19] - Centered Newton method for linear programming: Interior and 'exterior' point method' (in Japanese), in New Methods for Linear Programming 3, K. Tone, ed., The Institute of Statistical Mathematics, Tokyo, Japan, 1990, pp. 98-100.

[20] Y. YE, $A n O\left(n^{3} L\right)$ potential reduction algorithm for linear programming, Mathematical Programming, 50 (1991), pp. 239-258.

[21] Y. Ye, M. J. TOdD, AND S. Mizuno, An $O(\sqrt{n} L)$-iteration homogeneous and self-dual linear programming algorithm, Mathematics of Operations Research, 19 (1994), pp. 53-67.

[22] Y. ZHANG, On the convergence of a class of infeasible interior-point methods for the horizontal linear complementarity problem, SIAM Journal on Optimization, 4 (1994), pp. 208-227. 